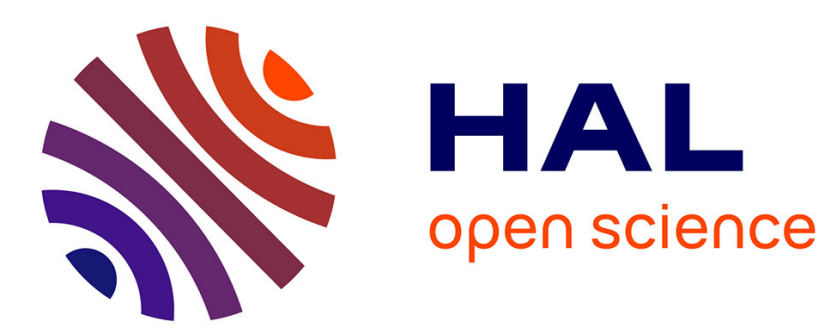

\title{
Homogenization of a nonlocal elastohydrodynamic lubrication problem: a new free boundary model
}

Guy Bayada, Sébastien Martin, Carlos Vazquez

\section{To cite this version:}

Guy Bayada, Sébastien Martin, Carlos Vazquez. Homogenization of a nonlocal elastohydrodynamic lubrication problem: a new free boundary model. 2004. hal-00008081v2

\section{HAL Id: hal-00008081 \\ https://hal.science/hal-00008081v2}

Preprint submitted on 15 Feb 2006

HAL is a multi-disciplinary open access archive for the deposit and dissemination of scientific research documents, whether they are published or not. The documents may come from teaching and research institutions in France or abroad, or from public or private research centers.
L'archive ouverte pluridisciplinaire HAL, est destinée au dépôt et à la diffusion de documents scientifiques de niveau recherche, publiés ou non, émanant des établissements d'enseignement et de recherche français ou étrangers, des laboratoires publics ou privés. 


\title{
HOMOGENIZATION OF A NONLOCAL ELASTOHYDRODYNAMIC LUBRICATION PROBLEM: A NEW FREE BOUNDARY MODEL*
}

\author{
G. Bayada \\ MAPLY CNRS UMR-5585 / LAMCOS CNRS UMR-5514, bat L. de Vinci, INSA de Lyon \\ 69621 Villeurbanne Cedex, France \\ guy.bayada@insa-lyon.fr \\ S. Martin \\ MAPLY CNRS UMR-5585, bat L. de Vinci, INSA de Lyon \\ 69621 Villeurbanne Cedex, France \\ sebastien.martin@insa-lyon.fr \\ C. Vázquez \\ Departamento de Matemáticas, Facultad de Informática, \\ Universidade da Coruña, Campus Elviña, 15071-A Coruña, España \\ carlosv@udc.es
}

\begin{abstract}
The present paper deals with the homogenization of a lubrication problem, using the periodic unfolding method. We study in particular the Reynolds-Hertz model, which takes into account elastohydrodynamic deformations of the upper surface, when highly oscillating roughness effects occur. The difficulty arises when considering cavitation free boundary phenomena, leading to highly nonlinear and nonlocal problems.

Keywords: Elastohydrodynamic lubrication; piezoviscous fluid; Elrod-Adams model; homogenization; two-scale convergence; numerical simulation.
\end{abstract}

AMS Subject Classification: 76D08, 35B40, 35J85, 76M50, 76M10

\section{Statement of the problem}

The greatly increasing number of industrial technical devices involving the presence of lubricated contacts motivates interest in studying more suitable models for the practical situations. Considering an elastic rolling ball or cylinder and a rigid plane leads to an elastohydrodynamic lubrication problem, taking into account the possibility of the ball/cylinder deformation. From a practical point of view, the introduction of surface periodic roughness during manufacturing processes cannot be avoided. In this rough elastohydrodynamic contact setting, it is important to state adequate mathematical models in order to perform the numerical simulation of the

* June 2004 
devices. For this, one possible tool is provided by the homogenization technique analyzed in the present paper.

The mathematical model, to be further detailed later, is governed by the following set of highly coupled and nonlinear equations:

$$
\begin{aligned}
& \nabla \cdot\left(h[p]^{3} e^{-\alpha p} \nabla p\right)=\frac{\partial}{\partial x_{1}}(\theta h[p]) \\
& p \geq 0, \quad 0 \leq \theta \leq 1, \quad p(1-\theta)=0,
\end{aligned}
$$

where $h[p]$, which is the effective gap between two close surfaces, contains a given rigid contribution $h_{r}$ and an elastic one, which strongly depends on the main unknown $p$ (lubricant pressure) in the following nonlocal form:

$$
h[p]=h_{r}+\int_{\Omega} k(\cdot, z) p(z) d z,
$$

the kernel $k$ depending on the kind of contact. Moreover, the fluid saturation, $\theta$, is related to the pressure by means of the Heaviside multivalued operator $H$ and the exponential term takes into account piezoviscous effects.

Basic aspects of the early developped elastohydrodynamic theory have been stated by Dowson and Higginson [12], where the three main common features of this kind of problems are already quoted: the fluid hydrodynamic displacement, the solid elastic deformation and the air bubble generation. Thus, the Reynolds equation, linear Hertz contact theory and different cavitation models try to mathematically analyse these three phenomena. Moreover, the modification of the initial fluid viscosity due to the presence of sufficiently high values of lubricant pressure might have to be taken into account, so that the complete modelling is extended to piezoviscous fluids. Thus the complete model takes into account the following aspects:

- The Reynolds equation has been used for a long time to describe the behaviour of a viscous flow between two close surfaces in relative motion [24]. It can be written as:

$$
\nabla \cdot\left(\frac{h_{r}^{3}}{6 \mu} \nabla p\right)=v_{0} \frac{\partial}{\partial x_{1}}\left(h_{r}\right)
$$

where $p$ is the pressure distribution, $h_{r}$ the gap between the two surfaces, $\mu$ the lubricant viscosity and $v_{0}$ the speed of the lower surface (the upper surface is assumed to be fixed). The transition of the Stokes equation to the Reynolds equation has been proved by Bayada and Chambat in [4].

- However, the earlier equation does not take into account cavitation phenomena: cavitation is defined as the rupture of the continuous film due to the formation of air bubbles and makes the Reynolds equation no longer valid in the cavitation area. In order to make it possible, various models have been used, the most popular perhaps being variational inequalities which have a strong mathematical basis but lack physical evidence. Thus, we use the Elrod-Adams model, which introduces the hypothesis that the 
cavitation region is a fluid-air mixture and an additional unknown $\theta$ (the saturation of fluid in the mixture) (see $[16,3]$ ).

Actually, elastohydrodynamic lubrication (EHL) occurs between point or line contact, so all the loading is concentrated over a small contact area. Typical applications are rolling element bearings, most gears, and cams and tappets [12]. The concentrated contact results in high peak pressures of 1-2 GPa between the surfaces. This is too high to be supported by a normal hydrodynamic film, and application of simple hydrodynamic theory predicts negligible oil film thickness. In practice films are formed and have thickness comparable to the surface roughness of normal gear and bearing materials. This is because the high pressure has two beneficial effects unaccounted for in hydrodynamics. Firstly, elastic flattening of the contacting surfaces occurs. Secondly, the high pressure greatly increases the viscosity of the lubricant in the contact. Elastohydrodynamic lubrication is consequently analysed using a combination of Reynold's equation, elasticity theory (the Hertz equation) and a lubricant viscosity-pressure equation. Thus, introducing the elastic deformation of the surfaces due to the fluid pressure and assuming the Hertzian contact theory for a parameter regime that corresponds to low speeds, low viscosity at ambient pressure or small elastic modulus, the effective gap is, in fact, linked to the pressure. Let us mention that piezoviscous properties of the fluid have to be taken into account in realistic applications. Thus, the viscosity is no longer constant and also depends on the pressure [2].

The mathematical analysis of different elastohydrodynamic problems taking into account the previous quoted features has been treated in the literature for the variational inequality cavitation model $[17,19,23,25]$ and for the Elrod-Adams model $[7,13]$.

The effect of surfaces periodic roughness on the behaviour of hydrodynamic and elastohydrodynamic magnitudes has been treated in numerous works. Some of the theoretical studies also include numerical examples which show how significant pressure and deformation perturbations appear due to the presence of surface asperities, either in the hydrodynamic regime $[8,9,20]$ or in the elastohydrodynamic one [6]. For the particular point and linear elastohydrodynamic contacts here treated, although some numerical methods have been proposed in the literature [18], the rigorous mathematical analysis to justify the homogenized models has not been performed yet. Furthermore, in this paper, the more realistic Elrod-Adams model is considered instead of the variational inequality one [6]. Notice that for the numerical simulation of micro-elastohydrodynamic contacts, the statement of well justified homogenized problems prevents from using extremely high numbers of mesh points to accurately compute the involved physical magnitudes (when directly solving the small parameter dependent problems).

Now, we present the full lubrication model, including cavitation phenomena and 
piezoviscous elastohydrodynamic aspects.

\section{Mathematical formulation of the EHL problem}

We consider a rectangular domain $\Omega=]-l_{1}, l_{1}[\times]-l_{2}, l_{2}\left[; \Gamma_{\star}\right.$ denotes the left vertical boundary and $\Gamma=\partial \Omega \backslash \Gamma_{\star}$ (see for instance FIG.1).

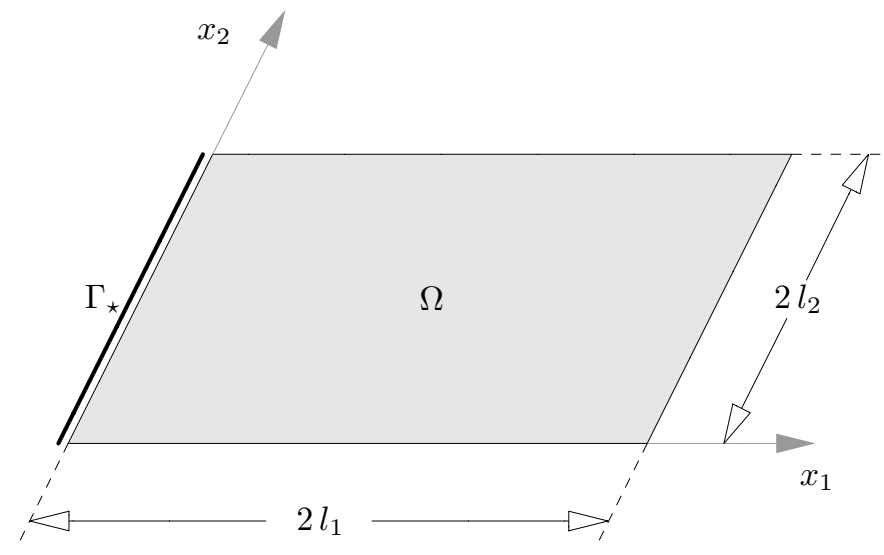

Fig. 1. Domain

We suppose that the following assumptions are satisfied.

Assumption 1.1 (Rigid contribution to the gap). The classical approximation of the rigid gap (see [12]) is given by the expression

$$
h_{r}(x)= \begin{cases}h_{0}+\frac{x_{1}^{2}+x_{2}^{2}}{2 R}, & \text { for ball bearings } \\ h_{0}+\frac{x_{1}^{2}}{R}, & \text { for linear bearings }\end{cases}
$$

that represents a parabolic approximation for a given sphere-plane (point contact) or cylinder-plane (line contact) gap, $R$ being the sphere or cylinder section radius.

Remark 1.1. The positive constant $h_{0}$ corresponds to the gap at the point nearest to contact. Clearly, the condition

$$
0<h_{0} \leq h_{r}(x) \leq h_{1}, \quad \text { with } h_{0}, h_{1} \text { two constants }
$$

is satisfied in the bounded domain $\Omega$.

As previously mentioned, in the Hertz theory, the effective gap is linked to the pressure through the following relationship

Assumption 1.2 (Deformable contribution to the gap). The effective gap between the surfaces is given by

$$
h[p](x)=h_{r}(x)+\int_{\Omega} k(x, z) p(z) d z, \quad \forall x \in \Omega
$$


where $h_{r}$ satisfies Assumption 1.1, and $k(x, z)$ is

$$
k(x, z)= \begin{cases}c_{0} \log \left|\frac{c_{1}-z_{1}}{x_{1}-z_{1}}\right|, & \text { for line contacts } \\ \frac{c_{0}}{\sqrt{\left(x_{1}-z_{1}\right)^{2}+\left(x_{2}-z_{2}\right)^{2}}}, & \text { for point contacts }\end{cases}
$$

where $c_{0}>0$ and $c_{1} \geq \max \left\{\left|x_{1}\right|, x \in \bar{\Omega}\right\}$.

Remark 1.2. Clearly $k$ is a positive function and there exists $\tilde{K}>0$ such that

$$
\|k(x, .)\|_{L^{1}(\Omega)} \leq \tilde{K}
$$

uniformly with respect to $x$. Let us notice that the expression of $h$ contains a rigid term, $h_{r}$, and an additional term due to the surface deformation.

Finally let us take into account the piezoviscous properties of the lubricant, i.e. the viscosity is no longer constant.

Assumption 1.3 (Piezoviscosity law). The viscosity obeys the Barus law [2]:

$$
\mu=\mu_{0} e^{\alpha p}
$$

where $\alpha \geq 0$ and $\mu_{0}>0$ denote the piezoviscosity constant and the zero pressure viscosity repectively.

The strong formulation of the problem is:

$$
\begin{aligned}
& x \in \Omega^{+}:\left\{\begin{array}{l}
\nabla \cdot\left(\frac{h^{3}[p](x)}{6 \mu_{0}} e^{-\alpha p(x)} \nabla p(x)\right)=v_{0} \frac{\partial}{\partial x_{1}}(\theta(x) h[p](x)) \\
p(x)>0 \text { and } \theta(x)=1
\end{array}\right. \\
& x \in \Omega_{0}:\left\{\begin{array}{l}
v_{0} \frac{\partial}{\partial x_{1}}(\theta(x) h[p](x))=0 \\
p(x)=0 \text { and } 0 \leq \theta(x) \leq 1
\end{array}\right. \\
& x \in \Sigma:\left\{\begin{array}{l}
\frac{h^{3}[p](x)}{6 \mu_{0}} e^{-\alpha p(x)} \frac{\partial p(x)}{\partial n}=v_{0}(1-\theta(x)) h[p](x) \cos (\vec{n}, \vec{i}) \\
p(x)=0
\end{array}\right.
\end{aligned}
$$

with the boundary conditions

$$
\begin{array}{ll}
v_{0} \theta h[p]-\frac{h^{3}[p]}{6 \mu_{0}} e^{-\alpha p} \frac{\partial p}{\partial n}=v_{0} \theta_{\star} h[p] & \text { on } \Gamma_{\star} \\
p=0 & \text { on } \Gamma
\end{array}
$$

where $v_{0}$ denotes the velocity of the lower surface in the $x_{1}$ direction, $\theta_{\star}$ is a supply parameter belonging to $[0,1], \vec{n}$ represents the unit normal vector to $\Sigma$ pointing to $\Omega_{0}, \vec{i}$ is the unit vector in the $x_{1}$ direction; and the sets appearing earlier are given 
by

$$
\begin{aligned}
& \Omega^{+}=\{x \in \Omega, p(x)>0\} \\
& \Omega_{0}=\{x \in \Omega, p(x)=0\} \\
& \Sigma=\partial \Omega^{+} \cup \Omega \quad \text { (cavitated region), } \\
& \Sigma \quad \text { (free boundary). }
\end{aligned}
$$

Thus, working in dimensionless data $\left(6 \mu_{0} v_{0}=1\right.$ for instance), the piezoviscous elastohydrodynamic problem can be written as

$$
\left(\mathcal{P}_{\theta}\right)\left\{\begin{array}{l}
\text { Find }(p, \theta) \in V \times L^{\infty}(\Omega) \text { such that: } \\
\int_{\Omega} h^{3}[p] e^{-\alpha p} \nabla p \nabla \phi=\int_{\Omega} \theta h[p] \frac{\partial \phi}{\partial x_{1}}+\int_{\Gamma_{\star}} \theta_{\star} h[p] \phi, \quad \forall \phi \in V \\
p \geq 0, \quad p(1-\theta)=0, \quad 0 \leq \theta \leq 1, \quad \text { a.e. },
\end{array}\right.
$$

where the functional space $V$ is defined as $V=\left\{v \in H^{1}(\Omega), v_{\mid \Gamma}=0\right\}$.

The boundary data, $\theta_{\star}$, satisfies:

\section{Assumption 1.4 (Saturation on the boundary).}

(i) $\theta_{\star} \in L^{\infty}\left(\Gamma_{\star}\right)$,

(ii) $0 \leq \theta_{\star}(z) \leq 1$, for a.e. $z \in \Gamma_{\star}$.

Finally, let us consider the following technical assumption.

Assumption 1.5 (Technical hypothesis on the data). The Sobolev exponent $r^{\star}>2$, the Sobolev constant $C(\Omega)$ (the norm of the trace mapping from $H^{1}(\Omega)$ to $\left.L^{2}\left(\Gamma_{\star}\right)\right)$ and the problem parameters satisfy the condition

$$
\frac{\alpha e C(\Omega)}{h_{0}^{2}}|\Omega|^{(1 / 2)-\left(1 / r^{\star}\right)} 2^{r^{\star} /\left(r^{\star}-2\right)} \leq 1,
$$

We have the following existence theorem:

Theorem 1.1 (Durany, García, Vázquez [13]). Under Assumptions 1.1-1.5, problem $\left(\mathcal{P}_{\theta}\right)$ admits at least a solution $(p, \theta)$ satisfying the following estimates:

$$
\|p\|_{H^{1}(\Omega)} \leq C_{1} \text { and }\|p\|_{L^{\infty}(\Omega)} \leq C_{2}
$$

where $C_{1}$ and $C_{2}$ depend on $\alpha, h_{0}, h_{1}, \widetilde{K}, \theta_{\star}, \Omega, \Gamma_{\star}, r^{\star}$.

Remark 1.3. The complete proof is given by Durany, García and Vázquez [13]. It is based on the introduction of a penalized problem and Schauder fixed-point theorem. We point out the fact that the earlier estimates are not a priori estimates. Thus, we cannot guarantee that each solution of the problem satisfies the earlier estimates. Assumption 1.5 guarantees an existence result if $\alpha$ is small enough: it holds if the physical configuration is not too far from the isoviscous case.

Remark 1.4. Other boundary conditions might be taken into account: a similar result has been proved with Dirichlet conditions on the pressure by Bayada, El Alaoui 
and Vázquez [7]. The homogenization study that follows can be easily adapted to this specific type of boundary conditions.

The next sections deal with homogenization of the lubrication problem, using the periodic unfolding method which has been introduced Cioranescu, Damlamian and Griso [11]. This method has strong links with the two-scale convergence technique which was introduced by Nguetseng [22], and further developped by Allaire [1], Lukkassen, Nguetseng and Wall [21].

\section{Homogenization of the EHL problem}

In this section, we state some preliminary results before the homogenization process of the lubrication problem. Let us introduce the microscale domain $Y=] 0,1[\times] 0,1[$. The nominal gap, i.e. without elastic deformation, is now described by the nominal regular thickness $h_{r}$ to which one must add the roughness defaults around the average gap. Thus, we consider that the nominal gap is described by:

$$
h_{r}^{\varepsilon}(x)=h_{r}(x)+\lambda\left(\frac{x}{\varepsilon}\right),
$$

where $h_{r}$ has been defined in Assumption 1.1 and $\lambda$ satisfies:

Assumption 2.1 (Roughness pattern).

(i) $\lambda \in C_{\sharp}(Y)=\left\{v \in C^{0}(Y)\right.$, $v$ is $Y$ periodic $\}$,

(ii) $\exists \lambda_{\max }>0, \quad\|\lambda\|_{L^{\infty}(Y)} \leq \lambda_{\max }<h_{0}$.

Remark 2.1. Assumptions 1.1 and 2.1 guarantee the uniform coerciveness (with respect to the parameter $\varepsilon$ ) of the bilinear form; in fact, we have

$$
\forall x \in \Omega, \quad 0<h_{0}-\|\lambda\|_{L^{\infty}(Y)} \leq h_{r}^{\varepsilon}(x) \leq h_{1}+\|\lambda\|_{L^{\infty}(Y)} .
$$

Let us remark that it leads us to consider the roughness of the upper surface, assumed to be fixed, so that the $x$ variable becomes highly oscillating. Thus it means that only the rigid contribution to the gap is rough.

Now let us define the effective gaps:

Definition 2.1. For any $q \in L^{\infty}(\Omega)$, let $h[q]$ and $h_{\varepsilon}[q]$ be the functions defined by:

$$
\begin{aligned}
h[q]: \Omega \times Y & \longrightarrow \mathbb{R} \\
(x, y) & \longrightarrow h[q](x, y)=h_{r}(x)+\lambda(y)+\int_{\Omega} k(x, z) q(z) d z, \\
h_{\varepsilon}[q]: \quad \Omega & \longrightarrow \mathbb{R} \\
x & \longrightarrow h_{\varepsilon}[q](x)=h[q]\left(x, \frac{x}{\varepsilon}\right) .
\end{aligned}
$$


Thus, we introduce the rough problem:

$\left(\mathcal{P}_{\theta}^{\varepsilon}\right)\left\{\begin{array}{l}\text { Find }\left(p_{\varepsilon}, \theta_{\varepsilon}\right) \in V \times L^{\infty}(\Omega) \text { such that: } \\ \int_{\Omega} h_{\varepsilon}^{3}\left[p_{\varepsilon}\right] e^{-\alpha p_{\varepsilon}} \nabla p_{\varepsilon} \nabla \phi=\int_{\Omega} \theta_{\varepsilon} h_{\varepsilon}\left[p_{\varepsilon}\right] \frac{\partial \phi}{\partial x_{1}}+\int_{\Gamma_{\star}} \theta_{\star} h_{\varepsilon}\left[p_{\varepsilon}\right] \phi, \quad \forall \phi \in V \\ p_{\varepsilon} \geq 0, \quad p_{\varepsilon}\left(1-\theta_{\varepsilon}\right)=0, \quad 0 \leq \theta_{\varepsilon} \leq 1, \quad \text { a.e. }\end{array}\right.$

In order to get an existence theorem, we adapt the assumptions to the rough problem. Therefore, Assumption 1.5 is replaced by:

Assumption 2.2. The Sobolev exponent $r^{\star}>2$, the Sobolev constant $C(\Omega)$ (the norm of the trace mapping from $H^{1}(\Omega)$ to $\left.L^{2}\left(\Gamma_{\star}\right)\right)$ and the problem parameters satisfy the condition

$$
\frac{\alpha e C(\Omega)}{\left(h_{0}-\|\lambda\|_{L^{\infty}(\Omega)}\right)^{2}}|\Omega|^{(1 / 2)-\left(1 / r^{\star}\right)} 2^{r^{\star} /\left(r^{\star}-2\right)} \leq 1 .
$$

Thus we get:

Theorem 2.1. Under Assumptions 1.1-1.4, 2.1 and 2.2, for any $\varepsilon>0$, problem $\left(\mathcal{P}_{\theta}^{\varepsilon}\right)$ admits at least a solution $\left(p_{\varepsilon}, \theta_{\varepsilon}\right)$ satisfying the following estimates:

$$
\left\|\nabla p_{\varepsilon}\right\|_{L^{2}(\Omega)} \leq C_{3}, \quad\left\|p_{\varepsilon}\right\|_{L^{\infty}(\Omega)} \leq C_{4}, \quad\left\|\theta_{\varepsilon}\right\|_{L^{\infty}(\Omega)} \leq 1
$$

where $C_{3}$ and $C_{4}$ only depend on $\alpha, h_{0}-\|\lambda\|_{L^{\infty}(\Omega)}, h_{1}, \widetilde{K}, \theta_{\star}, \Omega, \Gamma_{\star}, r^{\star}$.

Remark 2.2. In mechanical applications (ball or linear bearings), typical roughness is assumed to be either transverse or longitudinal. However, such an assumption on the roughness form is not necessary and more general shapes may be introduced.

Our purpose is to discuss the behaviour of problem $\left(\mathcal{P}_{\theta}^{\varepsilon}\right)$ when $\varepsilon$ goes to 0 , using periodi unfolding methods. From now on, we suppose that Assumptions 1.1-1.4, 2.1 and 2.2 are satisfied, in particular in Subsections 2.2-2.5.

\subsection{Preliminaries to the periodic unfolding method}

First we recall some useful definitions and results for the periodic unfolding method [11].

Lemma 2.1. The separable Banach space $L^{2}\left(\Omega ; C_{\sharp}(Y)\right)$ is dense in $L^{2}(\Omega \times Y)$. Moreover, if $f \in L^{2}\left(\Omega ; C_{\sharp}(Y)\right)$, then $x \mapsto \sigma_{\varepsilon}(f)(x)=f(x, x / \varepsilon)$ is a measurable function such that

$$
\left\|\sigma_{\varepsilon}(f)\right\|_{L^{2}(\Omega)} \leq\|f\|_{L^{2}\left(\Omega ; C_{\sharp}(Y)\right)} .
$$

Let us now briefly introduce periodic unfolding methods [11]: 
Definition 2.2 (Unfolding operator). For any $x \in \mathbb{R}^{2}$, let be $[x]_{Y}$ the unique integer combination in $\mathbb{Z}^{2}$ such that $x-[x]_{Y}$ belongs to $Y$. We also define $\{x\}_{Y}=$ $x-[x]_{Y} \in Y$, so that, for each $x \in \mathbb{R}^{2}$, one has

$$
x=\varepsilon\left(\left[\frac{x}{\varepsilon}\right]_{Y}+\left\{\frac{x}{\varepsilon}\right\}_{Y}\right) .
$$

Then the unfolding operator $\mathcal{T}_{\varepsilon}: L^{2}(\Omega) \rightarrow L^{2}(\Omega \times Y)$ is defined as follows: for $w \in L^{2}(\Omega)$, extended by zero outside $\Omega$,

$$
\mathcal{T}_{\varepsilon}(w)(x, y)=w\left(\varepsilon\left[\frac{x}{\varepsilon}\right]_{Y}+\varepsilon y\right), \quad \text { for } x \in \Omega \text { and } y \in Y .
$$

Let us now recall Propositions 1 and 2 of Cioranescu, Damlamian and Griso [11]:

Proposition 2.1. One has the following integration formula

$$
\int_{\Omega} w=\int_{\Omega \times Y} \mathcal{T}_{\bar{\varepsilon}}(w), \quad \forall w \in L^{1}(\Omega) .
$$

Proposition 2.2. Let $\left\{u_{\varepsilon}\right\}$ be a bounded sequence in $L^{2}(\Omega)$. Then the following propositions are equivalent:

(i) $\mathcal{T}_{\varepsilon}\left(u_{\varepsilon}\right)$ weakly converges to $u_{0}$ in $L^{2}(\Omega \times Y)$.

(ii) $u_{\varepsilon}$ two-scale converges to $u_{0}$.

Similarly to the two-scale convergence technique, the following properties hold:

\section{Lemma 2.2 .}

(i) Let $u_{\varepsilon}$ be a bounded sequence in $L^{2}(\Omega)$. Then there exists $u_{0} \in L^{2}(\Omega \times Y)$ such that, up to a subsequence, $\mathcal{T}_{\varepsilon}\left(u_{\varepsilon}\right)$ weakly converges to $u_{0}$ in $L^{2}(\Omega \times Y)$.

(ii) Let $u_{\varepsilon}$ be a bounded sequence in $H^{1}(\Omega)$, which weakly converges to a limit $u_{0} \in H^{1}(\Omega)$. Then $\mathcal{T}_{\varepsilon}\left(u_{\varepsilon}\right)$ weakly converges to $u_{0}$ in $L^{2}(\Omega \times Y)$ and there exists a function $u_{1} \in L^{2}\left(\Omega ; H_{\sharp}^{1}(Y) / \mathbb{R}\right)$ such that, up to a subsequence, $\mathcal{T}_{\varepsilon}\left(\nabla u_{\varepsilon}\right)$ weakly converges to $\nabla u_{0}+\nabla_{y} u_{1}$ in $L^{2}(\Omega \times Y)$.

To conclude this brief introduction, we have the following (straightforward) property:

Proposition 2.3. Let $u \in L^{2}(\Omega)$. If a sequence $u_{\varepsilon} \in L^{2}(\Omega)$ strongly converges to $u$ in $L^{2}(\Omega)$, then $\mathcal{T}_{\varepsilon}\left(u_{\varepsilon}\right)$ strongly converges to $u$ in $L^{2}(\Omega \times Y)$.

\subsection{Convergence results for problem $\left(\mathcal{P}_{\theta}^{\varepsilon}\right)$}

Lemma 2.3. There exists $p_{0} \in V$ such that, up to a subsequence:

$$
p_{\varepsilon} \rightarrow p_{0} \text { in } H^{1}(\Omega) \quad \text { and } \quad p_{\varepsilon} \rightarrow p_{0} \text { in } L^{2}(\Omega) .
$$

We have also the following convergences: 
(i) $\mathcal{T}_{\varepsilon}\left(p_{\varepsilon}\right)$ strongly converges to $p_{0}$ in $L^{2}(\Omega \times Y)$. Moreover, there exists a function $p_{1} \in L^{2}\left(\Omega ; H_{\sharp}^{1}(Y) / \mathbb{R}\right)$ and a subsequence $\varepsilon^{\prime}$, still denoted $\varepsilon$, such that $\mathcal{T}_{\varepsilon}\left(\nabla p_{\varepsilon}\right)$ weakly converges to $\nabla p_{0}+\nabla_{y} p_{1}$ in $L^{2}(\Omega \times Y)$.

(ii) There exists $\theta_{0} \in L^{2}(\Omega \times Y)$ and a subsequence $\varepsilon$ ", still denoted $\varepsilon$, such that $\mathcal{T}_{\varepsilon}\left(\theta_{\varepsilon}\right)$ weakly converges to $\theta_{0}$ in $L^{2}(\Omega \times Y)$.

Moreover, $p_{0} \geq 0$ a.e.

Proof. The convergence results are the consequence of Estimates 2.3 (see Theorem 2.1), which do not depend on $\varepsilon$, and Lemma 2.2.

Remark 2.3. The following proposition has been stated in the hydrodynamic case (see [9]). The same proof can be used, but we propose an alternate proof, based on periodic unfolding methods. However, the idea remains the same.

Proposition 2.4. $0 \leq \theta_{0} \leq 1$ and $p_{0}\left(1-\theta_{0}\right)=0$ a.e.

\section{Proof.}

- 1st step - As $0 \leq \theta_{\varepsilon}$ a.e. and using the definition of the unfolding operator, one has that $0 \leq \mathcal{T}_{\varepsilon}\left(\theta_{\varepsilon}\right)$ a.e. By Proposition 2.2, one knows that $\mathcal{T}_{\varepsilon}\left(\theta_{\varepsilon}\right)$ weakly converges to $\theta_{0}$ in $L^{2}(\Omega \times Y)$. Thus one has

$$
\int_{\Omega \times Y} \mathcal{T}_{\varepsilon}\left(\theta_{\varepsilon}\right) \phi \longrightarrow \int_{\Omega \times Y} \theta_{0} \phi, \quad \forall \phi \in L^{2}(\Omega \times Y) .
$$

We rewrite $\theta_{0}$ as $\theta_{0}=\theta_{0}^{+}-\theta_{0}^{-}$(with $w^{+}=\max (w, 0)$ and $w^{-}=-\min (w, 0)$, for any $w \in L^{2}(\Omega \times Y)$ ). Then using $\theta_{0}^{-}$as a test function in Equation (2.4):

$$
A_{\varepsilon}=\int_{\Omega \times Y} \mathcal{T}_{\varepsilon}\left(\theta_{\varepsilon}\right) \theta_{0}^{-} \longrightarrow-\int_{\Omega \times Y}\left(\theta_{0}^{-}\right)^{2}=A \leq 0 .
$$

Since $\mathcal{T}\left(\theta_{\varepsilon}\right) \geq 0$ a.e., $A_{\varepsilon}$ is a sequence of positive numbers converging to a non positive number. Then $A=0$ and $\theta_{0}^{-}=0$ a.e. The same method is used to prove $0 \leq 1-\theta_{0}$ a.e.

2nd step - The result is also easily obtained using periodic unfolding methods. Indeed by Lemma 2.3 and Proposition 2.2,

$$
\begin{aligned}
& \mathcal{T}_{\varepsilon}\left(p_{\varepsilon}\right) \rightarrow p_{0}, \text { in } L^{2}(\Omega \times Y), \\
& \mathcal{T}_{\varepsilon}\left(\theta_{\varepsilon}\right) \rightarrow \theta_{0}, \text { in } L^{2}(\Omega \times Y) .
\end{aligned}
$$

Thus one gets:

$$
\int_{\Omega \times Y} \mathcal{T}_{\varepsilon}\left(p_{\varepsilon}\left(1-\theta_{\varepsilon}\right)\right)=\int_{\Omega \times Y} \mathcal{T}_{\varepsilon}\left(p_{\varepsilon}\right) \mathcal{T}_{\varepsilon}\left(1-\theta_{\varepsilon}\right) \longrightarrow \int_{\Omega \times Y} p_{0}\left(1-\theta_{0}\right) .
$$

Since $p_{\varepsilon}\left(1-\theta_{\varepsilon}\right)=0$, passing to the limit gives

$$
\int_{\Omega \times Y} p_{0}\left(1-\theta_{0}\right)=0
$$


Moreover since $p_{0} \geq 0$ a.e. and $1-\theta_{0} \geq 0$ a.e. (see Lemma 2.3 and the first part of Proposition 2.4), the proof is concluded.

Lemma 2.4. For $1 \leq p \leq+\infty$,

(i) $\int_{\Omega} k(\cdot, z) p_{\varepsilon}(z) d z$ strongly converges to $\int_{\Omega} k(\cdot, z) p_{0}(z) d z$ in $L^{p}(\Omega)$,

(ii) $\int_{\Omega} k(\cdot, z) p_{\varepsilon}(z) d z$ strongly converges to $\int_{\Omega} k(\cdot, z) p_{0}(z) d z$ in $L^{p}\left(\Gamma_{\star}\right)$.

\section{Proof.}

Proof of $(i)$ - Since $k$ satisfies Equation (1.4), one gets by Lemma 1 of Oden and $\mathrm{Wu}[23]$ :

$$
\max _{x \in \bar{\Omega}}\left|\int_{\Omega} k(x, z)\left(p_{\varepsilon}(z)-p_{0}(z)\right) d z\right| \leq C_{5}(k)\left\|p_{\varepsilon}-p_{0}\right\|_{L^{q}(\Omega)}
$$

for any $q$ which can be written as $q=(2-s) /(1-s)>2$ with $0<s<1$. Moreover by Theorem IX.16 (Rellich-Kondrachov) in [10], one has $H^{1}(\Omega) \hookrightarrow L^{r}(\Omega)$, $\forall r \in\left[1,+\infty\left[\right.\right.$. Since $p_{\varepsilon} \rightarrow p_{0}$ in $H^{1}(\Omega)$ (see Lemma 2.3), then $p_{\varepsilon} \rightarrow p_{0}$ in $L^{q}(\Omega)$ and

$$
\left\|\int_{\Omega} k(\cdot, z)\left(p_{\varepsilon}(z)-p_{0}(z)\right) d z\right\|_{L^{\infty}(\Omega)} \longrightarrow 0
$$

At last, we gain (for $1 \leq p<+\infty$ )

$$
\left\|\int_{\Omega} k(\cdot, z)\left(p_{\varepsilon}(z)-p_{0}(z)\right) d z\right\|_{L^{p}(\Omega)} \leq|\Omega|^{1 / p}\left\|\int_{\Omega} k(\cdot, z)\left(p_{\varepsilon}(z)-p_{0}(z)\right) d z\right\|_{L^{\infty}(\Omega)},
$$

the result is proved.

- Proof of $(i i)$ - For $p=+\infty$, the result is immediatly obtained from Inequality $(2.5)$.

For $1 \leq p<+\infty$, let us compute $u_{\varepsilon}=\left\|\int_{\Omega} k(\cdot, z)\left(p_{\varepsilon}(z)-p_{0}(z)\right) d z\right\|_{L^{p}\left(\Gamma_{\star}\right)}$.

$$
\begin{aligned}
u_{\varepsilon} & =\left(\int_{\Gamma_{\star}}\left[\int_{\Omega} k(s, z)\left(p_{\varepsilon}(z)-p_{0}(z)\right) d z\right]^{p} d s\right)^{1 / p} \\
& \leq\left|\Gamma_{\star}\right|^{1 / p} \max _{x \in \bar{\Omega}}\left|\int_{\Omega} k(\cdot, z)\left(p_{\varepsilon}(z)-p_{0}(z)\right) d z\right|,
\end{aligned}
$$

and using Inequality (2.5) gives

$$
u_{\varepsilon} \leq\left|\Gamma_{\star}\right|^{1 / p} C_{5}(k)\left\|p_{\varepsilon}-p_{0}\right\|_{L^{q}(\Omega)},
$$

so that $u_{\varepsilon}$ exists and tends to 0 . 
Now we analyse the convergence of each term of problem $\left(\mathcal{P}_{\theta}^{\varepsilon}\right)$ :

Lemma 2.5. One has the following strong convergences in $L^{2}(\Omega \times Y)$ :

(i) $\mathcal{T}_{\varepsilon}\left(h_{\varepsilon}\left[p_{\varepsilon}\right]\right) \longrightarrow h\left[p_{0}\right]$,

(ii) $\mathcal{T}_{\varepsilon}\left(h_{\varepsilon}^{3}\left[p_{\varepsilon}\right]\right) \longrightarrow h^{3}\left[p_{0}\right]$,

(iii) $\mathcal{T}_{\varepsilon}\left(e^{-\alpha p_{\varepsilon}}\right) \longrightarrow e^{-\alpha p_{0}}$.

Moreover, as a direct consequence of (ii) and (iii), one has:

(iv) $\mathcal{T}_{\varepsilon}\left(h_{\varepsilon}^{3}\left[p_{\varepsilon}\right] e^{-\alpha p_{\varepsilon}}\right) \longrightarrow h^{3}\left[p_{0}\right] e^{-\alpha p_{0}}$.

\section{Proof.}

- Proof of $(i)-\mathcal{T}_{\varepsilon}\left(h_{\varepsilon}^{3}\left[p_{\varepsilon}\right]\right)$ strongly converges to $h^{3}\left[p_{0}\right]$ in $L^{2}(\Omega \times Y)$. Indeed, using the definition and linearity of the unfolding operator, we have:

$$
\mathcal{T}_{\varepsilon}\left(h_{\varepsilon}\left[p_{\varepsilon}\right]\right)(x, y)=h_{r}(x, y)+\mathcal{T}_{\varepsilon}\left(\int_{\Omega} k(x, z) p_{\varepsilon}(z) d z\right) .
$$

Using the property of the Hertz kernel (especially Lemma 2.4 and Proposition $2.3)$, we easily state that

$$
\mathcal{T}_{\varepsilon}\left(\int_{\Omega} k(x, z) p_{\varepsilon}(z) d z\right) \longrightarrow \int_{\Omega} k(x, z) p_{0}(z) d z, \quad \text { in } L^{2}(\Omega \times Y) .
$$

Proof of $(i i)$ - Obviously, $\mathcal{T}_{\varepsilon}\left(h_{\varepsilon}\left[p_{\varepsilon}\right]\right)$ and $h\left[p_{0}\right]$ have the following $L^{\infty}$ bound:

$$
C_{6}=3\left(h_{1}+\|\lambda\|_{L^{\infty}(\Omega)}+\widetilde{K} C_{4}\right)^{2},
$$

which does not depend on $\varepsilon$. Now, using the definition of the unfolding operator, we have:

$$
\int_{\Omega \times Y}\left(\mathcal{T}_{\varepsilon}\left(h_{\varepsilon}^{3}\left[p_{\varepsilon}\right]\right)-h^{3}\left[p_{0}\right]\right)^{2} \leq 9 C_{6}^{4} \int_{\Omega \times Y}\left(\mathcal{T}_{\varepsilon}\left(h_{\varepsilon}\left[p_{\varepsilon}\right]\right)-h\left[p_{0}\right]\right)^{2}
$$

which tends to 0 , by the result stated at $(i)$.

- Proof of (iii) - By Proposition 2.3, it is sufficient to prove that $e^{-\alpha p_{\varepsilon}}$ strongly converges to $e^{-\alpha p_{0}}$ in $L^{2}(\Omega)$. For this, let us point out the fact that $z \mapsto e^{-\alpha z}$ is a Lipschitz continuous function on $\mathbb{R}^{+}, \alpha$ being a Lipschitz constant. Thus, we have

$$
\int_{\Omega}\left(e^{-\alpha p_{\varepsilon}}-e^{-\alpha p_{0}}\right)^{2} \leq \alpha^{2} \int_{\Omega}\left(p_{\varepsilon}-p_{0}\right)^{2}
$$

which tends to 0 and concludes this proof.

- Proof of $(i v)$ - By using the two previous results, the convergence result is stated due to the fact that each term is bounded in $L^{\infty}(\Omega)$. 
Now, we are interested in the convergence of the boundary term:

Lemma 2.6. Let $\gamma$ denote the trace operator and let us define

$$
\widehat{h\left[p_{0}\right]}=\gamma\left(h_{r}(\cdot)+\int_{0}^{1} \lambda\left(\left(0, y_{2}\right)\right) d y_{2}+\int_{\Omega} k(\cdot, z) p_{0}(z) d z\right) .
$$

Then, one has:

$$
\gamma\left(h_{\varepsilon}\left[p_{\varepsilon}\right]\right) \rightarrow \widehat{h\left[p_{0}\right]} \text { in } L^{2}\left(\Gamma_{\star}\right) .
$$

Proof. In the boundary integral of problem $\left(\mathcal{P}_{\theta}^{\varepsilon}\right), h_{\varepsilon}\left[p_{\varepsilon}\right]$ has to be taken in the sense of traces. Thus, since we have

$$
h_{\varepsilon}\left[p_{\varepsilon}\right](x)=h_{r}(x)+\lambda\left(\frac{x}{\varepsilon}\right)+\int_{\Omega} k(x, z) p_{\varepsilon}(z) d z,
$$

it can be written as the sum of a function which belongs to $L^{\infty}\left(\Gamma_{\star}\right)$, namely

$$
\gamma\left(h_{r}(\cdot)+\int_{\Omega} k(\cdot, z) p_{\varepsilon}(z) d z\right),
$$

and the trace of the oscillating function $x \mapsto \sigma_{\varepsilon}(\lambda)(x)=\lambda(x / \varepsilon)$ (according to the definition of the operator $\sigma_{\varepsilon}$ given in Lemma 2.1), i.e.

$$
\gamma\left(\sigma_{\varepsilon}(\lambda)(\cdot)\right) \text {. }
$$

Thus, let us study the convergence of each term with respect to $\varepsilon$ :

- First, the following convergence holds:

$$
\gamma\left(h_{r}(\cdot)+\int_{\Omega} k(\cdot, z) p_{\varepsilon}(z) d z\right) \longrightarrow \gamma\left(h_{r}(\cdot)+\int_{\Omega} k(\cdot, z) p_{0}(z) d z\right) \text {, in } L^{2}\left(\Gamma_{\star}\right) .
$$

Indeed, by linearity, the difference of these two terms is equal to the trace of

$$
\int_{\Omega} k(\cdot, z)\left(p_{\varepsilon}(z)-p_{0}(z)\right) d z
$$

which strongly converges to 0 in $L^{2}\left(\Gamma_{\star}\right)$ by Lemma 2.4 .

- Next, using the assumptions on the roughness regularity, $\gamma\left(\sigma_{\varepsilon}(\lambda)\right)$ can be identified to the function $x_{2} \mapsto \lambda\left(\left(0, x_{2} / \varepsilon\right)\right)$, which weakly converges in $L^{2}(] 0,1[)$ to its average with respect to $y_{2}$, namely the constant

$$
\int_{0}^{1} \lambda\left(\left(0, y_{2}\right)\right) d y_{2}
$$

Thus the proof is achieved. 


\subsection{Homogenization of the EHL problem (general case)}

Once we have obtained the limits of the different terms which appear in problem $\left(\mathcal{P}_{\theta}^{\varepsilon}\right)$, we state, as usual with the two-scale convergence technique, the macroscopic and microscopic equations for the homogenized problem.

Proposition 2.5. The limit functions $p_{0}, p_{1}$ and $\theta_{0}$ satisfy the following equations:

- Macroscopic equation - For every $\phi$ in $V$,

$$
\int_{\Omega}\left(\int_{Y} h^{3}\left[p_{0}\right] e^{-\alpha p_{0}}\left[\nabla p_{0}+\nabla_{y} p_{1}\right]\right) \nabla \phi=\int_{\Omega}\left(\int_{Y} \theta_{0} h\left[p_{0}\right]\right) \frac{\partial \phi}{\partial x_{1}}+\int_{\Gamma_{\star}} \theta_{\star} \widehat{h\left[p_{0}\right]} \phi .
$$

- Microscopic equation - For a.e. $x \in \Omega$, for every $\psi \in H_{\sharp}^{1}(Y)$,

$$
\int_{Y} h^{3}\left[p_{0}\right](x, \cdot) e^{-\alpha p_{0}(x)}\left[\nabla p_{0}(x)+\nabla_{y} p_{1}(x, \cdot)\right] \nabla_{y} \psi=\int_{Y} \theta_{0}(x, \cdot) h\left[p_{0}\right](x, \cdot) \frac{\partial \psi}{\partial y_{1}} .
$$

Proof. Considering problem $\left(\mathcal{P}_{\theta}^{\varepsilon}\right)$, we have, using Proposition 2.1:

$$
\begin{aligned}
& \int_{\Omega \times Y} \mathcal{T}_{\varepsilon}\left(h_{\varepsilon}^{3}\left[p_{\varepsilon}\right]\right.\left.e^{-\alpha p_{\varepsilon}}\right) \mathcal{T}_{\varepsilon}\left(\nabla p_{\varepsilon}\right) \mathcal{T}_{\varepsilon}\left(\nabla \varphi_{\varepsilon}\right) \\
&=\int_{\Omega \times Y} \mathcal{T}_{\varepsilon}\left(\theta_{\varepsilon}\right) \mathcal{T}_{\varepsilon}\left(h_{\varepsilon}\left[p_{\varepsilon}\right]\right) \mathcal{T}_{\varepsilon}\left(\frac{\partial \varphi_{\varepsilon}}{\partial x_{1}}\right)+\int_{\Gamma^{\star}} \theta_{\star}(r) h_{\varepsilon}\left[p_{\varepsilon}\right](r) \varphi_{\varepsilon}(r) d r .
\end{aligned}
$$

for all $\varphi_{\varepsilon} \in V$. Taking a test function $x \mapsto \phi(x)$, with $\phi \in V$ and using the convergence results stated in Lemmas $2.3,2.5$ and 2.6 gives the macroscopic equation by passing to the limit with respect to $\varepsilon$. Now taking the test function $x \mapsto \varepsilon \phi(x) \psi(x / \varepsilon)$, with $\phi \in \mathcal{D}(\Omega)$ and $\psi \in H_{\sharp}^{1}(Y)$, gives the microscopic one.

Definition 2.3. For a given $p_{0} \in L^{\infty}(\Omega)$, let us define the following functions:

$$
\begin{aligned}
& a\left[p_{0}\right](x, y)=h^{3}\left[p_{0}\right](x, y),(x, y) \in \Omega \times Y, \\
& b\left[p_{0}\right](x, y)=h\left[p_{0}\right](x, y), \quad(x, y) \in \Omega \times Y .
\end{aligned}
$$

Let us define the local problems, respectively denoted $\left(\mathcal{M}_{i}^{\star}\right),\left(\mathcal{N}_{i}^{\star}\right)$ and $\left(\mathcal{N}_{i}^{0}\right)$ :

Find $W_{i}^{\star}, \chi_{i}^{\star}, \chi_{i}^{0}(i=1,2)$ in $L^{2}\left(\Omega ; H_{\sharp}^{1}(Y) / \mathbb{R}\right)$, such that, for a.e. $x \in \Omega$ :

$$
\begin{aligned}
\int_{Y} a\left[p_{0}\right](x, \cdot) \nabla_{y} W_{i}^{\star}(x, \cdot) \nabla_{y} \psi & =\int_{Y} a\left[p_{0}\right](x, \cdot) \frac{\partial \psi}{\partial y_{i}}, \\
\int_{Y} a\left[p_{0}\right](x, \cdot) \nabla_{y} \chi_{i}^{\star}(x, \cdot) \nabla_{y} \psi & =\int_{Y} b\left[p_{0}\right](x, \cdot) \frac{\partial \psi}{\partial y_{i}}, \\
\int_{Y} a\left[p_{0}\right](x, \cdot) \nabla_{y} \chi_{i}^{0}(x, \cdot) \nabla_{y} \psi & =\int_{Y} \theta_{0}(x, \cdot) b\left[p_{0}\right](x, \cdot) \frac{\partial \psi}{\partial y_{i}},
\end{aligned}
$$

for all $\psi \in H_{\sharp}^{1}(Y)$. We immediatly have the following proposition:

Proposition 2.6. The local problem $\left(\mathcal{M}_{i}^{\star}\right)\left(\right.$ resp. $\left.\left(\mathcal{N}_{i}^{\star}\right),\left(\mathcal{N}_{i}^{0}\right)\right)$ admits a unique solution $W_{i}^{\star}$ (resp. $\left.\chi_{i}^{\star}, \chi_{i}^{0}\right)$ in $L^{2}\left(\Omega ; H_{\sharp}^{1}(Y) / \mathbb{R}\right)$. 
Theorem 2.2. The homogenized problem can be written as:

$$
\left(\mathcal{P}_{\theta}^{\star}\right)\left\{\begin{array}{l}
\text { Find }\left(p_{0}, \Theta_{1}, \Theta_{2}\right) \in V \times L^{\infty}(\Omega) \times L^{\infty}(\Omega) \text { such that: } \\
\int_{\Omega} e^{-\alpha p_{0}} \mathcal{A}\left[p_{0}\right] \cdot \nabla p_{0} \nabla \phi=\int_{\Omega} \underline{b}^{0}\left[p_{0}\right] \nabla \phi+\int_{\Gamma_{\star}} \theta_{\star} \widehat{h\left[p_{0}\right]} \phi, \quad \forall \phi \in V \\
p_{0} \geq 0, \quad p_{0}\left(1-\Theta_{i}\right)=0,(i=1,2) \quad \text { a.e. }
\end{array}\right.
$$

with $\tilde{f}(x)=\int_{Y} f(x, y) d y$, and

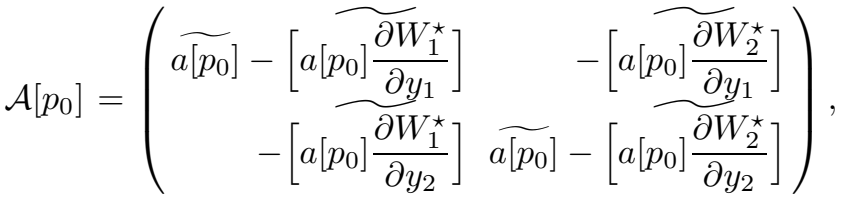

$$
\begin{aligned}
& \underline{b}^{0}\left[p_{0}\right]=\left(\begin{array}{cc}
\Theta_{1}\left[p_{0}\right] & b_{1}^{\star}\left[p_{0}\right] \\
\Theta_{2}\left[p_{0}\right] & b_{2}^{\star}\left[p_{0}\right]
\end{array}\right),
\end{aligned}
$$

with the notations $(i=1,2)$ :

$$
\left.b_{i}^{\star}\left[p_{0}\right]=\widetilde{b\left[p_{0}\right]}-\left[\widetilde{a\left[p_{0}\right] \frac{\partial \chi_{i}^{\star}}{\partial y_{i}}}\right], \quad b_{i}^{0}\left[p_{0}\right]=\widetilde{\left[\theta_{0} b\left[p_{0}\right]\right.}\right]-\left[\overline{a\left[p_{0}\right] \frac{\partial \chi_{i}^{0}}{\partial y_{i}}}\right],
$$

and defining the following ratios $(i=1,2)$ :

$$
\Theta_{i}\left[p_{0}\right]=\frac{b_{i}^{0}\left[p_{0}\right]}{b_{i}^{\star}\left[p_{0}\right]} .
$$

Proof. From the local problems, we easily obtain in $L^{2}\left(\Omega ; H_{\sharp}^{1}(Y) / \mathbb{R}\right)$ :

$$
p_{1}(x, y)=-\left(\begin{array}{l}
W_{1}^{\star}(x, y) \\
W_{2}^{\star}(x, y)
\end{array}\right) \cdot \nabla p_{0}(x)+e^{\alpha p_{0}(x)} \chi_{1}^{0}(x, y) .
$$

The homogenized problem follows by replacing the previous expression of $p_{1}$ in the macroscopic equation.

Remark 2.4. The homogenized lubrication problem can be considered as a generalized elastohydrodynamic Reynolds-type problem with two cavitation parameters $\Theta_{i}(i=1,2)$. Let us notice the fact that we do not have the property $0 \leq \Theta_{i} \leq 1$, i.e. we cannot guarantee that homogenized cavitation parameters are smaller than 1 in cavitation areas! Thus, at that point, the homogenized problem does not have a structure similar to the initial one. But, in the next subsections, we prove the following additional results:

- in Subsection 2.4, we state that, among the solutions of the homogenized problem, there exists a class of solutions with isotropic saturation, that is, the homogenized problem $\left(\mathcal{P}_{\theta}^{\star}\right)$ admits a solution $\left(p_{0}, \Theta, \Theta\right)$ with $p_{0} \geq 0$ and $p_{0}(1-\Theta)=0$ and also the additive property (which lacks in the formulation of the homogenized problem $\left(\mathcal{P}_{\theta}^{\star}\right)$ in the general case): $0 \leq \Theta \leq 1$ a.e. 
- in Subsection 2.5, we state that, under additional assumptions on the roughness pattern, only one single saturation function $\Theta$ appears in the homogenized problem. Moreover, it satisfies $0 \leq \Theta \leq 1$ a.e in $\Omega$.

\subsection{Existence of solutions with isotropic saturation}

This subsection is devoted to the proof of the following theorem:

Theorem 2.3. The homogenized problem $\left(\mathcal{P}_{\theta}^{\star}\right)$ admits a solution $\left(p_{0}, \Theta, \Theta\right)$ with the property $0 \leq \Theta \leq 1$ (and also $p_{0} \geq 0$, $p_{0}(1-\Theta)=0$ ) a.e.

Theorem 2.3 guarantees the existence of solutions with isotropic saturation $\Theta$. Moreover, the saturation satisfies the property $0 \leq \Theta \leq 1$, which lacks in the general formulation of the homogenized problem. The result is obtained in the following three steps which are based on the existence result and the corresponding method used in Durany, García, Vázquez [13]:

- 1st step: Introduction of a penalized problem,

- 2nd step: Homogenization of the penalized problem,

- 3rd step: Convergence with respect to the penalized parameter.

Remark 2.5. Interestingly, in the earlier scheme, forgetting the 2 nd step (i.e. omitting the homogenization step) would lead us to the existence result for problem $\left(\mathcal{P}_{\theta}^{\varepsilon}\right)$, namely Theorem 2.1. Thus, the reader should not be surprised to see that constants which have been already used or defined in Theorem 2.1 appear in the details of the forthcoming proof.

For convenience, these three steps are given in details and the idea of the proof is sketched at the end of this subsection. It can be noticed that the general frame is quite similar than in the hydrodynamic case developped in [9]. Nevertheless, it is much more difficult from a technical point of view, as it will be pointed out. In particular, the 3rd step needs further analysis due to the nonlocal term and the piezoviscous one.

- 1st step: Introduction of a penalized problem

As in the smooth case studied by Durany, García, Vázquez [13], we introduce the following $\varepsilon$ dependent penalized problem:

$\left(\mathcal{P}_{\eta}^{\varepsilon}\right)\left\{\begin{array}{l}\text { Find } p_{\varepsilon}^{\eta} \in V \text { such that: } \\ \int_{\Omega} h_{\varepsilon}^{3}\left[p_{\varepsilon}^{\eta}\right] e^{-\alpha p_{\varepsilon}^{\eta}} \nabla p_{\varepsilon}^{\eta} \nabla \phi=\int_{\Omega} H_{\eta}\left(p_{\varepsilon}^{\eta}\right) h_{\varepsilon}\left[p_{\varepsilon}^{\eta}\right] \frac{\partial \phi}{\partial x_{1}}+\int_{\Gamma_{\star}} \theta_{\star} h_{\varepsilon}\left[p_{\varepsilon}^{\eta}\right] \phi, \quad \forall \phi \in V \\ p_{\varepsilon}^{\eta} \geq 0, \quad \text { a.e., }\end{array}\right.$

where the function $H_{\eta}$ is the usual approximation of the Heaviside graph (see [9]). The application of Theorem 3.2. of Durany, García, Vázquez [13], which is based on a fixed point technique leads to the following results: 
Theorem 2.4. For every $\eta>0$, problem $\left(\mathcal{P}_{\eta}^{\varepsilon}\right)$ admits a positive solution. Moreover, we can obtain the following $(\varepsilon, \eta)$ independent estimates:

$$
\left\|p_{\varepsilon}^{\eta}\right\|_{H^{1}(\Omega)} \leq C_{3}, \quad\left\|p_{\varepsilon}^{\eta}\right\|_{L^{\infty}(\Omega)} \leq C_{4} .
$$

Remark 2.6. We point out the fact that Theorem 2.4 holds under Assumptions 1.1-1.4, 2.1 and 2.2 which are implicitely imposed as in previous subsections. In particular, Assumption 2.2 is necessary to allow the use of a fixed point technique and obtain Theorem 2.4. In particular, the $L^{\infty}$ estimates play an important role in the proof of existence of isotropic solutions.

2nd step: Homogenization of the penalized problem

We proceed to the homogenization of problem $\left(\mathcal{P}_{\eta}^{\varepsilon}\right)$ with respect to $\varepsilon$ : from Estimates (2.9) and using the periodic unfolding method (as in the previous subsection), we immediatly get the following convergence results and macro/microscopic decomposition (for convenience, proofs are omitted when they are close to the ones stated in previous subsections):

Proposition 2.7. There exists $p_{0}^{\eta} \in V$ ( $p_{0}^{\eta} \geq 0$ a.e. $), p_{1} \in L^{2}\left(\Omega ; H_{\sharp}(Y) / \mathbb{R}\right)$ such that, up to a subsequence,

(i) $p_{\varepsilon}^{\eta}$ weakly converges to $p_{0}^{\eta}$ in $H^{1}(\Omega)$,

(ii) $\mathcal{T}_{\varepsilon}\left(\nabla p_{\varepsilon}^{\eta}\right)$ weakly converges to $\nabla p_{0}^{\eta}+\nabla_{y} p_{1}^{\eta}$ in $L^{2}(\Omega \times Y)$.

Moreover, we have:

- Macroscopic equation - For every $\phi$ in $V$,

$$
\int_{\Omega}\left(\int_{Y} h^{3}\left[p_{0}^{\eta}\right] e^{-\alpha p_{0}^{\eta}}\left[\nabla p_{0}^{\eta}+\nabla_{y} p_{1}^{\eta}\right]\right) \nabla \phi=\int_{\Omega}\left(\int_{Y} H_{\eta}\left(p_{0}^{\eta}\right) h\left[p_{0}^{\eta}\right]\right) \frac{\partial \phi}{\partial x_{1}}+\int_{\Gamma_{\star}} \theta_{\star} \widehat{h\left[p_{0}^{\eta}\right]} \phi .
$$

- Microscopic equation - For a.e. $x \in \Omega$, for every $\psi \in H_{\sharp}^{1}(Y)$,

$$
\int_{Y} h^{3}\left[p_{0}^{\eta}\right](x, \cdot) e^{-\alpha p_{0}^{\eta}(x)}\left[\nabla p_{0}^{\eta}(x)+\nabla_{y} p_{1}^{\eta}(x, \cdot)\right] \nabla_{y} \psi=\int_{Y} H_{\eta}\left(p_{0}^{\eta}(x)\right) h\left[p_{0}^{\eta}\right](x, \cdot) \frac{\partial \psi}{\partial y_{1}} .
$$

Then, recalling the definition of $a[\cdot]$ and $b[\cdot]$ (see Definition 2.3), and introducing the local problems, respectively denoted $\left(\mathcal{M}_{i}^{\eta}\right),\left(\mathcal{N}_{i}^{\eta}\right)$ :

Find $W_{i}^{\eta}, \chi_{i}^{\eta}$ in $L^{2}\left(\Omega ; H_{\sharp}^{1}(Y) / \mathbb{R}\right)$, such that, for a.e. $x \in \Omega$ :

$$
\begin{aligned}
\int_{Y} a\left[p_{0}^{\eta}\right](x, \cdot) \nabla_{y} W_{i}^{\eta}(x, \cdot) \nabla_{y} \psi & =\int_{Y} a\left[p_{0}^{\eta}\right](x, \cdot) \frac{\partial \psi}{\partial y_{i}} \\
\int_{Y} a\left[p_{0}^{\eta}\right](x, \cdot) \nabla_{y} \chi_{i}^{\eta}(x, \cdot) \nabla_{y} \psi & =\int_{Y} b\left[p_{0}^{\eta}\right](x, \cdot) \frac{\partial \psi}{\partial y_{i}}
\end{aligned}
$$

for all $\psi \in H_{\sharp}^{1}(Y)$. We can state: 
Lemma 2.7. The homogenized penalized problem is

$$
\begin{aligned}
& \left(\mathcal{P}_{\eta}^{\star}\right)\left\{\begin{array}{l}
\text { Find } p_{0}^{\eta} \in V \text { such that: } \\
\int_{\Omega} e^{-\alpha p_{0}^{\eta}} \mathcal{A}^{\eta}\left[p_{0}^{\eta}\right] \cdot \nabla p_{0}^{\eta} \nabla \phi=\int_{\Omega} H_{\eta}\left(p_{0}^{\eta}\right) \underline{b}^{\eta}\left[p_{0}^{\eta}\right] \nabla \phi+\int_{\Gamma_{\star}} \theta_{\star} \widehat{h\left[p_{0}^{\eta}\right]} \phi, \quad \forall \phi \in V \\
p_{0}^{\eta} \geq 0, \quad \text { a.e., }
\end{array}\right. \\
& \text { with } \mathcal{A}^{\eta}\left[p_{0}^{\eta}\right]=\widetilde{a\left[p_{0}^{\eta}\right]} I-a\left[\widehat{\left.p_{0}^{\eta}\right] \nabla W^{\eta}} \text { and } \underline{b}^{\eta}\left[p_{0}^{\eta}\right]=\left(\begin{array}{r}
\widetilde{b\left[p_{0}^{\eta}\right]}-\left(\widetilde{a\left[p_{0}^{\eta}\right] \frac{\partial \chi_{1}^{\eta}}{\partial y_{1}}}\right) \\
-\left(a\left[p_{0}^{\eta}\right] \frac{\partial \chi_{1}^{\eta}}{\partial y_{2}}\right)
\end{array}\right)\right.
\end{aligned}
$$

Proof. The following equality in $L^{2}\left(\Omega ; H_{\sharp}^{1}(Y) / \mathbb{R}\right)$ is classically obtained using the local problems:

$$
p_{1}^{\eta}(x, y)=-W^{\eta}(x, y) \cdot \nabla p_{0}^{\eta}(x)+e^{\alpha p_{0}^{\eta}(x)} H_{\eta}\left(p_{0}^{\eta}(x)\right) \chi_{1}^{\eta}(x, y) .
$$

Using Equation (2.12) in the macroscopic equation gives us:

$$
\begin{aligned}
& \int_{\Omega} e^{-\alpha p_{0}^{\eta}} \widetilde{\left[a\left[p_{0}^{\eta}\right]\right.} I-a\left[\widetilde{\left.p_{0}^{\eta}\right] \nabla W^{\eta}}\right] \cdot \nabla p_{0}^{\eta} \nabla \phi \\
& =\int_{\Omega} H_{\eta}\left(\widetilde{\left.p_{0}^{\eta}\right)} \widetilde{b\left[p_{0}^{\eta}\right]}-\left(\widetilde{a\left[p_{0}^{\eta}\right]} \frac{\partial \chi_{1}^{\eta}}{\partial y_{1}}\right)\right] \frac{\partial \phi}{\partial x_{1}}+\int_{\Omega} H_{\eta}\left(p_{0}^{\eta}\right)\left[-\left(a\left[p_{0}^{\eta}\right] \frac{\partial \chi_{1}^{\eta}}{\partial y_{2}}\right)\right] \frac{\partial \phi}{\partial x_{2}} \\
& +\int_{\Gamma_{\star}} \theta_{\star} \widehat{h\left[p_{0}^{\eta}\right]} \phi,
\end{aligned}
$$

for every $\phi \in V$. Then, the proof is concluded.

3rd step: Behaviour of the homogenized penalized problem with respect to $\eta$

Now we study the behaviour of the homogenized penalized problem when $\eta$ tends to 0.

Proposition 2.8. There exists $p_{0} \in V$ and $\Theta \in L^{\infty}(\Omega)$ such that

$$
p_{0}^{\eta} \rightarrow p_{0} \text { in } H^{1}(\Omega), \quad H_{\eta}\left(p_{0}^{\eta}\right) \rightarrow \Theta \text { in } L^{\infty}(\Omega) \text { weak-ᄎ. }
$$

Moreover, $p_{0} \geq 0,0 \leq \Theta \leq 1$ and $p_{0}(1-\Theta)=0$ a.e.

Proof. The convergences only come from estimates satisfied by $p_{0}^{\eta}$ (see Estimates $(2.9))$ :

$$
\left\|p_{0}^{\eta}\right\|_{H^{1}(\Omega)} \leq C_{3}, \quad\left\|p_{0}^{\eta}\right\|_{L^{\infty}(\Omega)} \leq C_{4}
$$

The properties and relationships between $p_{0}$ and $\Theta$ are classically obtained as in the smooth problem.

Now we state:

Proposition 2.9. $e^{-\alpha p_{0}^{\eta}} \mathcal{A}^{\eta}\left[p_{0}^{\eta}\right]$ strongly converges to $e^{-\alpha p_{0}} \mathcal{A}\left[p_{0}\right]$ in $L^{2}(\Omega)$. 
Proof. We prove the result in three steps:

(a) $\left\|\mathcal{A}^{\eta}\left[p_{0}^{\eta}\right]\right\|_{L^{\infty}(\Omega)} \leq \widetilde{C},\left\|\mathcal{A}\left[p_{0}\right]\right\|_{L^{\infty}(\Omega)} \leq \widetilde{C}$, where the constant $\widetilde{C}$ does not depend on $\eta$,

(b) $\mathcal{A}^{\eta}\left[p_{0}^{\eta}\right] \longrightarrow \mathcal{A}\left[p_{0}\right]$, a.e.,

(c) $e^{-\alpha p_{0}^{\eta}} \mathcal{A}^{\eta}\left[p_{0}^{\eta}\right]$ strongly converges to $e^{-\alpha p_{0}} \mathcal{A}\left[p_{0}\right]$ in $L^{2}(\Omega)$.

- Proof of $(a)$ :

Let us recall that $\mathcal{A}^{\eta}\left[p_{0}^{\eta}\right]=\widetilde{a\left[p_{0}^{\eta}\right]} I-a\left[\widetilde{\left.p_{0}^{\eta}\right] \nabla_{y} W^{\eta}}\right.$. Obviously, we have $\widetilde{a\left[p_{0}^{\eta}\right]} \leq C_{7}$ with

$$
C_{7}=\left(h_{1}+\|\lambda\|_{L^{\infty}(\Omega)}+\widetilde{K} C_{4}\right)^{3} .
$$

Thus, we just have to state the estimates for terms of the form

$$
a\left[p_{0}^{\eta}\right] \frac{\partial W_{i}^{\eta}}{\partial y_{k}}, \quad(i, k=1,2) .
$$

Using $W_{i}^{\eta}$ as a test function in the variational formulation of problem $\left(\mathcal{M}_{i}^{\eta}\right)$ (see Equation (2.10)) gives for a.e. $x \in \Omega$

$$
\int_{Y} a\left[p_{0}^{\eta}\right](x, \cdot)\left|\nabla_{y} W_{i}^{\eta}(x, \cdot)\right|^{2}=\int_{Y} a\left[p_{0}^{\eta}\right](x, \cdot) \frac{\partial W_{i}^{\eta}}{\partial y_{i}}(x, \cdot) .
$$

Then, in the left-hand side, we write $a\left[p_{0}^{\eta}\right]$ as $a^{2}\left[p_{0}^{\eta}\right] / a\left[p_{0}^{\eta}\right]$ and use a lower bound of $1 / a\left[p_{0}^{\eta}\right]$, that is

$$
\frac{1}{C_{7}} \int_{Y}\left|a\left[p_{0}^{\eta}\right](x, \cdot) \nabla_{y} W_{i}^{\eta}(x, \cdot)\right|^{2} \leq \int_{Y} a\left[p_{0}^{\eta}\right](x, \cdot)\left|\nabla_{y} W_{i}^{\eta}(x, \cdot)\right|^{2}
$$

and this, together with Inequality (2.13), gives

$$
\int_{Y}\left|a\left[p_{0}^{\eta}\right](x, \cdot) \nabla_{y} W_{i}^{\eta}(x, \cdot)\right|^{2} \leq|Y| C_{7}^{2},
$$

which means

$$
\left\|a\left[p_{0}^{\eta}\right](x, \cdot) \frac{\partial W_{i}^{\eta}}{\partial y_{k}}(x, \cdot)\right\|_{L^{2}(Y)}^{2} \leq|Y| C_{7}^{2}, \quad(i, k=1,2) .
$$

Then, using the Cauchy-Schwarz inequality,

$$
\left|\int_{Y} a\left[p_{0}^{\eta}\right](x, \cdot) \frac{\partial W_{i}^{\eta}}{\partial y_{k}}(x, \cdot)\right| \leq|Y|^{1 / 2}\left\|a\left[p_{0}^{\eta}\right](x, \cdot) \frac{\partial W_{i}^{\eta}}{\partial y_{k}}(x, \cdot)\right\|_{L^{2}(Y)}
$$

that is,

$$
\left|a\left[p_{0}^{\eta}\right] \frac{\partial W_{i}^{\eta}}{\partial y_{k}}(x)\right| \leq|Y| C_{7}, \quad(i, k=1,2) .
$$


Let us remark that Inequality (2.14) holds for a.e. $x \in \Omega$ so that the matrix

$$
a\left[\widehat{\left.p_{0}^{\eta}\right] \nabla_{y} W^{\eta}}\right.
$$

is bounded in $L^{\infty}(\Omega)$ by a constant which does not depend on $\eta$. Thus

$$
\mathcal{A}^{\eta}\left[p_{0}^{\eta}\right]=\widetilde{a\left[p_{0}^{\eta}\right]} I-a\left[\widehat{\left.p_{0}^{\eta}\right] \nabla_{y}} W^{\eta}\right.
$$

is bounded by a constant $\widetilde{C}$ which does not depend on $\eta$. With the same method, as $p_{0}$ satisfies $\left\|p_{0}\right\|_{H^{1}(\Omega)} \leq C_{3},\left\|p_{0}\right\|_{L^{\infty}(\Omega)} \leq C_{4}$, one proves that

$$
\mathcal{A}\left[p_{0}\right]=\widetilde{a\left[p_{0}\right]} I-a\left[\widetilde{\left.p_{0}\right] \nabla_{y}} W^{\star}\right.
$$

is also bounded by $\widetilde{C}$.

- Proof of $(b)$ :

Let us recall that, with the same arguments that have been used in the proof of Lemma 2.4 (see Inequality (2.5)), one has:

$$
\int_{\Omega} k(\cdot, z) p_{0}^{\eta}(z) d z \longrightarrow \int_{\Omega} k(\cdot, z) p_{0}(z) d z \text { in } L^{\infty}(\Omega) .
$$

In particular, the convergence holds almost everywhere. Let be $x_{0}$ such that:

$$
\int_{\Omega} k\left(x_{0}, z\right) p_{0}^{\eta}(z) d z \longrightarrow \int_{\Omega} k\left(x_{0}, z\right) p_{0}(z) d z .
$$

Recalling local problem $\left(\mathcal{M}_{i}^{\eta}\right)$, one has,

$$
\int_{Y} a\left[p_{0}^{\eta}\right]\left(x_{0}, \cdot\right) \nabla_{y} W_{i}^{\eta}\left(x_{0}, \cdot\right) \nabla_{y} \psi=\int_{Y} a\left[p_{0}^{\eta}\right]\left(x_{0}, \cdot\right) \frac{\partial \psi}{\partial y_{i}}, \quad \forall \psi \in H_{\sharp}^{1}(Y) .
$$

Using $W_{i}^{\eta}\left(x_{0}, \cdot\right)$ as a test function and using upper and lower bounds of $a\left[p_{0}^{\eta}\right]$ gives:

$$
\left\|\nabla W_{i}^{\eta}\left(x_{0}, \cdot\right)\right\|_{L^{2}(Y)} \leq C_{7} / C_{8}
$$

with $C_{8}=\left(h_{0}-\|\lambda\|_{L^{\infty}(\Omega)}\right)^{3}$. Thus, $W_{i}^{\eta}\left(x_{0}, \cdot\right)$ is bounded in $H_{\sharp}^{1}(Y) / \mathbb{R}$ by a constant which does not depend on $\eta$. Then, there exists $F_{i}\left(x_{0}, \cdot\right) \in H_{\sharp}^{1}(Y) / \mathbb{R}$ such that $\nabla_{y} W_{i}^{\eta}\left(x_{0}, \cdot\right)$ weakly converges to $\nabla_{y} F_{i}\left(x_{0}, \cdot\right)$ in $L^{2}(Y)$. Moreover, since $a\left[p_{0}^{\eta}\right]\left(x_{0}, \cdot\right)$ strongly converges to $a\left[p_{0}\right]\left(x_{0}, \cdot\right)$ in $L^{2}(Y)$, one has for every $\psi \in H_{\sharp}^{1}(Y)$ :

$$
\begin{aligned}
\int_{Y} a\left[p_{0}^{\eta}\right]\left(x_{0}, \cdot\right) \nabla_{y} W_{i}^{\eta}\left(x_{0}, \cdot\right) \nabla_{y} \psi & \longrightarrow \int_{Y} a\left[p_{0}\right]\left(x_{0}, \cdot\right) \nabla_{y} F_{i}\left(x_{0}, \cdot\right) \nabla_{y} \psi \\
\int_{Y} a\left[p_{0}^{\eta}\right]\left(x_{0}, \cdot\right) \frac{\partial \psi}{\partial y_{i}} & \longrightarrow \int_{Y} a\left[p_{0}\right]\left(x_{0}, \cdot\right) \frac{\partial \psi}{\partial y_{i}}
\end{aligned}
$$

Thus, we get for every $\psi \in H_{\sharp}^{1}(Y)$ :

$$
\int_{Y} a\left[p_{0}\right]\left(x_{0}, \cdot\right) \nabla_{y} F_{i}\left(x_{0}, \cdot\right) \nabla_{y} \psi=\int_{Y} a\left[p_{0}\right]\left(x_{0}, \cdot\right) \frac{\partial \psi}{\partial y_{i}}
$$


and, by uniqueness of the solution to the local problem,

$$
\begin{aligned}
& W_{i}^{\star}\left(x_{0}, \cdot\right)=F_{i}\left(x_{0}, \cdot\right) \text {, in } H_{\sharp}^{1}(Y) / \mathbb{R}, \\
& \nabla_{y} W_{i}^{\star}\left(x_{0}, \cdot\right)=\nabla_{y} F_{i}\left(x_{0}, \cdot\right) \text {, in } L^{2}(Y) \text {. }
\end{aligned}
$$

Now, since we have:

$$
\begin{aligned}
a\left[p_{0}^{\eta}\right]\left(x_{0}, \cdot\right) & \rightarrow a\left[p_{0}\right]\left(x_{0}, \cdot\right), \text { in } L^{2}(Y), \\
\nabla_{y} W_{i}^{\eta}\left(x_{0}, \cdot\right) & \rightarrow \nabla_{y} W_{i}^{\star}\left(x_{0}, \cdot\right), \text { in } L^{2}(Y),
\end{aligned}
$$

we easily deduce that:

$$
\int_{Y} a\left[p_{0}^{\eta}\right]\left(x_{0}, y\right) \nabla_{y} W_{i}^{\eta}\left(x_{0}, y\right) d y \longrightarrow \int_{Y} a\left[p_{0}\right]\left(x_{0}, y\right) \nabla_{y} W_{i}^{\star}\left(x_{0}, y\right) d y,
$$

that is,

$$
\left[a [ \widetilde { p _ { 0 } ^ { \eta } ] \nabla _ { y } } W _ { i } ^ { \eta } ] ( x _ { 0 } ) \longrightarrow \left[a\left[\widetilde{\left.p_{0}\right] \nabla_{y} W_{i}^{\star}}\right]\left(x_{0}\right) .\right.\right.
$$

Since it is clear that $\widetilde{a\left[p_{0}^{\eta}\right]}\left(x_{0}\right)$ converges to $\widetilde{a\left[p_{0}\right]}\left(x_{0}\right)$, one has:

$$
\widetilde{a\left[p_{0}^{\eta}\right]}\left(x_{0}\right)-\left[a\left[\widetilde{\left.p_{0}^{\eta}\right] \nabla_{y}} W_{i}^{\eta}\right]\left(x_{0}\right) \longrightarrow \widetilde{a\left[p_{0}\right]}\left(x_{0}\right)-\left[a \widetilde{a\left[p_{0}\right] \nabla_{y} W_{i}^{\star}}\right]\left(x_{0}\right),\right.
$$

which states the result (b).

Proof of $(c)$ :

Let us denote $f^{\eta}=\mathcal{A}^{\eta}\left[p_{0}^{\eta}\right], f=\mathcal{A}\left[p_{0}\right]$ and $g^{\eta}=\left(f^{\eta}-f\right)^{2}$. It is clear that:

- $\left\|g^{\eta}\right\|_{L^{\infty}(\Omega)} \leq 4 \widetilde{C}^{2}$ (see property $(a)$ ),

○ $g^{\eta} \longrightarrow 0$ a.e. (see property $(b)$ ).

Thus, by the Lebesgue theorem, $\mathcal{A}^{\eta}\left[p_{0}^{\eta}\right]$ strongly converges to $\mathcal{A}\left[p_{0}\right]$ in $L^{2}(\Omega)$. Now let us denote

$$
r_{\eta}=\int_{\Omega}\left(e^{-\alpha p_{0}^{\eta}} \mathcal{A}^{\eta}\left[p_{0}^{\eta}\right]-e^{-\alpha p_{0}} \mathcal{A}\left[p_{0}\right]\right)^{2} .
$$

Then, we have:

$$
\begin{aligned}
r_{\eta} & =\int_{\Omega}\left(\left(e^{-\alpha p_{0}^{\eta}}-e^{-\alpha p_{0}}\right) \mathcal{A}^{\eta}\left[p_{0}^{\eta}\right]+e^{-\alpha p_{0}}\left(\mathcal{A}^{\eta}\left[p_{0}^{\eta}\right]-\mathcal{A}\left[p_{0}\right]\right)\right)^{2} \\
& \leq 2 \int_{\Omega}\left(e^{-\alpha p_{0}^{\eta}}-e^{-\alpha p_{0}}\right)^{2}\left(\mathcal{A}^{\eta}\left[p_{0}^{\eta}\right]\right)^{2}+2 \int_{\Omega}\left(e^{-\alpha p_{0}}\right)^{2}\left(\mathcal{A}^{\eta}\left[p_{0}^{\eta}\right]-\mathcal{A}\left[p_{0}\right]\right)^{2} .
\end{aligned}
$$

Since $\left\|\mathcal{A}^{\eta}\left[p_{0}^{\eta}\right]\right\|_{L^{\infty}(\Omega)} \leq \widetilde{C}$ (see property $(a)$ ) and $\left\|e^{-\alpha p_{0}}\right\|_{L^{\infty}(\Omega)} \leq 1$, one has:

$$
r_{\eta} \leq 2 \widetilde{C}^{2} \int_{\Omega}\left(e^{-\alpha p_{0}^{\eta}}-e^{-\alpha p_{0}}\right)^{2}+2 \int_{\Omega}\left(\mathcal{A}^{\eta}\left[p_{0}^{\eta}\right]-\mathcal{A}\left[p_{0}\right]\right)^{2} .
$$

Since $x \mapsto e^{-\alpha x}$ is $\alpha$-Lipschitz continuous on $\mathbb{R}^{+}$, one has

$$
r_{\eta} \leq 2 \widetilde{C}^{2} \alpha^{2} \int_{\Omega}\left(p_{0}^{\eta}-p_{0}\right)^{2}+2 \int_{\Omega}\left(\mathcal{A}^{\eta}\left[p_{0}^{\eta}\right]-\mathcal{A}\left[p_{0}\right]\right)^{2} .
$$


Then, as $p_{0}^{\eta}$ (resp. $\left.\mathcal{A}^{\eta}\left[p_{0}^{\eta}\right]\right)$ strongly converges to $p_{0}\left(\right.$ resp. $\left.\mathcal{A}\left[p_{0}\right]\right)$ in $L^{2}(\Omega), r_{\eta}$ tends to 0 , which concludes the proof.

Proposition 2.10. $\underline{b}^{\eta}\left[p_{0}^{\eta}\right]$ strongly converges to $\underline{b}^{\star}\left[p_{0}\right]$ in $L^{2}(\Omega)$.

Proof. The proof is similar to the one of Proposition 2.9. More precisely, we state

(a) $\left\|\underline{b}^{\eta}\left[p_{0}^{\eta}\right]\right\|_{L^{\infty}(\Omega)} \leq \widetilde{D},\left\|\underline{b}^{\star}\left[p_{0}\right]\right\|_{L^{\infty}(\Omega)} \leq \widetilde{D}$, where $\widetilde{D}$ does not depend on $\eta$,

(b) $\underline{b}^{\eta}\left[p_{0}^{\eta}\right] \longrightarrow \underline{b}^{\star}\left[p_{0}\right]$ a.e.,

and the proof is concluded with the Lebesgue theorem.

Proposition 2.11. $\widehat{h\left[p_{0}^{\eta}\right]}$ strongly converges to $\widehat{h\left[p_{0}\right]}$ in $L^{2}\left(\Gamma_{\star}\right)$.

Proof. The proof is straightforward from the properties of the Hertz kernel (see, in particular, Lemma 2.4).

Now we conclude this subsection by the proof of Theorem 2.3:

Proof of Theorem 2.3. By Lemma 2.7, there exists $p_{0}^{\eta} \in V$ such that

$$
\int_{\Omega} e^{-\alpha p_{0}^{\eta}} \mathcal{A}^{\eta}\left[p_{0}^{\eta}\right] \cdot \nabla p_{0}^{\eta} \nabla \phi=\int_{\Omega} H_{\eta}\left(p_{0}^{\eta}\right) \underline{b}^{\eta}\left[p_{0}^{\eta}\right] \nabla \phi+\int_{\Gamma_{\star}} \theta_{\star} \widehat{h\left[p_{0}^{\eta}\right]} \phi, \quad \forall \phi \in V .
$$

Next, from Propositions 2.8 and 2.9, we have:

$$
\begin{aligned}
& \nabla p_{0}^{\eta} \quad \rightarrow \quad \nabla p_{0}, \quad \text { in } L^{2}(\Omega), \\
& e^{-\alpha p_{0}^{\eta}} \mathcal{A}^{\eta}\left[p_{0}^{\eta}\right] \rightarrow e^{-\alpha p_{0}} \mathcal{A}\left[p_{0}\right] \text {, in } L^{2}(\Omega) \text {, }
\end{aligned}
$$

so that

$$
\int_{\Omega} e^{-\alpha p_{0}^{\eta}} \mathcal{A}^{\eta}\left[p_{0}^{\eta}\right] \cdot \nabla p_{0}^{\eta} \nabla \phi \longrightarrow \int_{\Omega} e^{-\alpha p_{0}} \mathcal{A}\left[p_{0}\right] \cdot \nabla p_{0} \nabla \phi, \quad \forall \phi \in V .
$$

Moreover, from Propositions 2.8 and 2.10, we have:

$$
\begin{array}{ll}
H_{\eta}\left(p_{0}^{\eta}\right) \rightarrow \Theta, & \text { in } L^{\infty}(\Omega) \text { weak- }, \\
\underline{b}^{\eta}\left[p_{0}^{\eta}\right] \rightarrow \underline{b}^{\star}\left[p_{0}\right], & \text { in } L^{2}(\Omega),
\end{array}
$$

so that

$$
\int_{\Omega} H_{\eta}\left(p_{0}^{\eta}\right) \underline{b}^{\eta}\left[p_{0}^{\eta}\right] \nabla \phi \longrightarrow \int_{\Omega} \Theta \underline{b}^{\star}\left[p_{0}\right] \nabla \phi, \quad \forall \phi \in V .
$$

Next, by Proposition 2.11, we obtain:

$$
\int_{\Gamma_{\star}} \theta_{\star} \widehat{h\left[p_{0}^{\eta}\right]} \phi \longrightarrow \int_{\Gamma_{\star}} \theta_{\star} \widehat{h\left[p_{0}\right]} \phi, \quad \forall \phi \in V .
$$

Thus, passing to the limit in the homogenized penalized problem, we get

$$
\int_{\Omega} e^{-\alpha p_{0}} \mathcal{A}\left[p_{0}\right] \cdot \nabla p_{0} \nabla \phi=\int_{\Omega} \Theta \underline{b}^{\star}\left[p_{0}\right] \nabla \phi+\int_{\Gamma_{\star}} \theta_{\star} \widehat{h\left[p_{0}\right]} \phi, \quad \forall \phi \in V,
$$


with $p_{0} \geq 0, p_{0}(1-\Theta)=0$ and $0 \leq \Theta \leq 1$ a.e. (by Proposition 2.8), and the proof is concluded.

Remark 2.7. An interesting point is to consider that, in the homogenized problem, we are not able to identify an "equivalent gap" since anisotropic effects classically appear in the coefficients. Nevertheless, Theorem 2.3 allows us to define not only one single saturation function $\Theta$ but also one single deformation

$$
\int_{\Omega} k(x, z) p_{0}(z) d z
$$

which is an important point in terms of mechanical applications.

\subsection{Particular cases}

In this subsection, due to particular choices of the roughness pattern, local problems have obvious analytical solutions (see [9]) so that it is possible to obtain self contained Reynolds equations for $p_{0}$ and one single saturation function $\Theta$.

Theorem 2.5 (Transverse roughness). If $\lambda$ does not depend on $y_{2}$, the homogenized problem $\left(\mathcal{P}_{\theta}^{\star}\right)$ is

$$
\left\{\begin{array}{l}
\text { Find }\left(p_{0}, \Theta\right) \in V \times L^{\infty}(\Omega) \text { such that: } \\
\int_{\Omega} e^{-\alpha p_{0}} \mathcal{A}\left[p_{0}\right] \cdot \nabla p_{0} \nabla \phi=\int_{\Omega} \Theta b^{\star}\left[p_{0}\right] \frac{\partial \phi}{\partial x_{1}}+\int_{\Gamma_{\star}} \theta_{\star} \widehat{h\left[p_{0}\right]} \phi, \quad \forall \phi \in V \\
p_{0} \geq 0, \quad p_{0}(1-\Theta)=0, \quad 0 \leq \Theta \leq 1, \quad \text { a.e., }
\end{array}\right.
$$

with the following homogenized coefficients:

$$
\mathcal{A}\left[p_{0}\right](x)=\left(\begin{array}{cc}
\widetilde{\frac{1}{h^{-3}\left[p_{0}\right](x)}} & 0 \\
0 & \widetilde{h^{3}\left[p_{0}\right]}(x)
\end{array}\right), \quad b^{\star}\left[p_{0}\right](x)=\frac{\widetilde{h^{-2}\left[p_{0}\right]}(x)}{\widehat{h^{-3}\left[p_{0}\right](x)}} .
$$

Moreover $\left(\mathcal{P}_{\theta}^{\star}\right)$ admits at least $\left(p_{0}, \Theta\right)$ as a solution where $\left(p_{0}, \theta_{0}\right)$ is the two-scale limit of $\left(p_{\varepsilon}, \theta_{\varepsilon}\right)$ (solution of problem $\left(\mathcal{P}_{\theta}^{\varepsilon}\right)$ ), and the link between the microscopic / macroscopic saturation function is given by:

$$
\Theta(x)=\left[\frac{1}{\widetilde{h^{-2}\left[p_{0}\right]}}\left(\widetilde{\theta_{0}}\right)\right](x) .
$$

Theorem 2.6 (Longitudinal roughness). If $\lambda$ does not depend on $y_{1}$, the homogenized problem $\left(\mathcal{P}_{\theta}^{\star}\right)$ is

$$
\left\{\begin{array}{l}
\text { Find }\left(p_{0}, \Theta\right) \in V \times L^{\infty}(\Omega) \text { such that: } \\
\int_{\Omega} e^{-\alpha p_{0}} \mathcal{A}\left[p_{0}\right] \cdot \nabla p_{0} \nabla \phi=\int_{\Omega} \Theta b^{\star}\left[p_{0}\right] \frac{\partial \phi}{\partial x_{1}}+\int_{\Gamma_{\star}} \theta_{\star} \widehat{h\left[p_{0}\right]} \phi, \quad \forall \phi \in V \\
p_{0} \geq 0, \quad p_{0}(1-\Theta)=0, \quad 0 \leq \Theta \leq 1, \quad \text { a.e., }
\end{array}\right.
$$


with the following homogenized coefficients:

$$
\mathcal{A}\left[p_{0}\right](x)=\left(\begin{array}{cc}
\widetilde{h^{3}\left[p_{0}\right]}(x) & 0 \\
0 & \frac{1}{\widetilde{h^{-3}\left[p_{0}\right]}(x)}
\end{array}\right), \quad b^{\star}\left[p_{0}\right](x)=\widetilde{h\left[p_{0}\right]}(x) .
$$

Moreover $\left(\mathcal{P}_{\theta}^{\star}\right)$ admits at least $\left(p_{0}, \Theta\right)$ as a solution where $\left(p_{0}, \theta_{0}\right)$ is the two-scale limit of $\left(p_{\varepsilon}, \theta_{\varepsilon}\right)$ (solution of problem $\left(\mathcal{P}_{\theta}^{\varepsilon}\right)$ ), and the link between the microscopic / macroscopic saturation function is given by:

$$
\Theta(x)=\frac{\left.\widetilde{\left(\theta_{0} h\left[p_{0}\right]\right.}\right)}{\widetilde{h\left[p_{0}\right]}}(x) .
$$

In the next section, we focus on numerical tests which illustrate the theoretical results which have been established in this section. In particular, we are interested in longitudinal and transverse roughness cases which have a great interest in terms of mechanical applications.

\section{Numerical examples}

In this section, the numerical simulation of micro-elastohydrodynamic contacts is performed to illustrate the theoretical results of convergence stated in the previous sections. For the numerical solution of the $\varepsilon$ dependent problems and their corresponding homogenized one, we propose an algorithm based on a fixed-point iteration between the hydrodynamic (Elrod-Adams) problem and the elastic (Hertz) one [14]. Furthermore, the hydrodynamic problem is solved using the characteristics method to deal with the convection term combined with a finite element spatial discretization and a duality method for the maximal monotone nonlinearity associated to the Elrod-Adams model. The elastic problem is approximated by using appropriate quadrature formulas in Equation (1.3). More precisely, the triangle edges midpoints are chosen as integration nodes. The combination of these numerical techniques has been already successfully used in previous papers dealing with the elastohydrodynamic related smooth problems $[14,15]$.

We adress the numerical simulation of dimensionless ball bearing contacts so that, for a domain $\Omega=]-2 l_{1}, l_{1}[\times]-l_{2}, l_{2}[$, we pose the problem

$$
\left\{\begin{array}{l}
\text { Find }\left(p_{\varepsilon}, \theta_{\varepsilon}\right) \in V \times L^{\infty}(\Omega) \text { such that: } \\
\int_{\Omega} h_{\varepsilon}^{3}\left[p_{\varepsilon}\right] e^{-\alpha p_{\varepsilon}} \nabla p_{\varepsilon} \nabla \phi=\int_{\Omega} \theta_{\varepsilon} h_{\varepsilon}\left[p_{\varepsilon}\right] \frac{\partial \phi}{\partial x_{1}}+\int_{\Gamma_{\star}} \theta_{\star} h_{\varepsilon}\left[p_{\varepsilon}\right] \phi, \quad \forall \phi \in V \\
p_{\varepsilon} \geq 0, \quad p_{\varepsilon}\left(1-\theta_{\varepsilon}\right)=0, \quad 0 \leq \theta_{\varepsilon} \leq 1, \quad \text { a.e. },
\end{array}\right.
$$

where the effective gap can be written as $h_{\varepsilon}\left[p_{\varepsilon}\right](x)=h_{r}^{\varepsilon}(x)+h_{d}\left[p_{\varepsilon}\right](x)$, the rigid 
and elastic contributions being given by

$$
\begin{aligned}
& h_{r}^{\varepsilon}(x)=h_{0}+\frac{x_{1}^{2}+x_{2}^{2}}{2}+\alpha_{1} \sin \left(\frac{6 l_{1} \pi\left(x_{1}+2 l_{1}\right)}{\varepsilon}\right)+\alpha_{2} \sin \left(\frac{4 l_{2} \pi\left(x_{2}+l_{2}\right)}{\varepsilon}\right), \\
& h_{d}\left[p_{\varepsilon}\right](x)=\frac{2}{\pi^{2}} \int \frac{p_{\varepsilon}(z)}{\sqrt{\left(x_{1}-z_{1}\right)^{2}+\left(x_{2}-z_{2}\right)^{2}}} d z
\end{aligned}
$$

and the following set of parameters:

- transverse case: $\quad l_{1}=2, \quad l_{2}=5, \quad h_{0}=0.5, \quad\left(\alpha_{1}, \alpha_{2}\right)=\left(0.5 h_{0}, 0\right)$,

- longitudinal case: $l_{1}=2, \quad l_{2}=2, \quad h_{0}=0.6, \quad\left(\alpha_{1}, \alpha_{2}\right)=\left(0,0.85 h_{0}\right)$.

Other parameters involved in the equation are taken to $\alpha=1$ (piezoviscosity parameter) and $\theta_{\star}=0.3$ (boundary data). The previous data have been taken from dimensionless equations associated to a small load imposed problem [15].

The homogenized problem, in the transverse or longitudinal case, can be written as

$$
\left\{\begin{array}{l}
\text { Find }\left(p_{0}, \Theta\right) \in V \times L^{\infty}(\Omega) \text { such that: } \\
\int_{\Omega} e^{-\alpha p_{0}}\left(\begin{array}{cc}
a_{1}\left[p_{0}\right] & 0 \\
0 & a_{2}\left[p_{0}\right]
\end{array}\right) \cdot \nabla p_{0} \nabla \phi=\int_{\Omega} \Theta b\left[p_{0}\right] \frac{\partial \phi}{\partial x_{1}}+\int_{\Gamma_{\star}} \theta_{\star} c\left[p_{0}\right] \phi, \forall \phi \in V \\
p_{0} \geq 0, \quad p_{0}(1-\Theta)=0, \quad 0 \leq \Theta \leq 1, \quad \text { a.e. }
\end{array}\right.
$$

In TABLE 1, we present the functional coefficients $a_{1}, a_{2}, b$ and $c$ that appear in the homogenized problem for purely transverse and purely longitudinal roughness cases which have been partially computed with MATHEMATICA Software Package.

\begin{tabular}{|c||c|c|}
\hline & Transverse roughness & Longitudinal roughness \\
\hline \hline$h[p](x, y)$ & $h_{r}(x)+h_{d}[p](x)+\alpha_{1} \sin \left(2 \pi y_{1}\right)$ & $h_{r}(x)+h_{d}[p](x)+\alpha_{2} \sin \left(2 \pi y_{2}\right)$ \\
\hline$a_{1}\left[p_{0}\right]$ & $2 \frac{\left(\left(h_{r}+h_{d}[p]\right)^{2}-\alpha_{1}^{2}\right)^{5 / 2}}{2\left(h_{r}+h_{d}[p]\right)^{2}+h_{r}^{2}}$ & $\left(h_{r}+h_{d}[p]\right)^{3}+\frac{3}{2}\left(h_{r}+h_{d}[p]\right) \alpha_{2}^{2}$ \\
\hline$a_{2}\left[p_{0}\right]$ & $\left(h_{r}+h_{d}[p]\right)^{3}+\frac{3}{2}\left(h_{r}+h_{d}[p]\right) \alpha_{1}^{2}$ & $2 \frac{\left(\left(h_{r}+h_{d}[p]\right)^{2}-\alpha_{2}^{2}\right)^{5 / 2}}{2\left(h_{r}+h_{d}[p]\right)^{2}+\alpha_{2}^{2}}$ \\
\hline$b\left[p_{0}\right]$ & $2\left(h_{r}+h_{d}[p]\right) \frac{\left(h_{r}+h_{d}[p]\right)^{2}-\alpha_{1}^{2}}{2\left(h_{r}+h_{d}[p]\right)^{2}+\alpha_{1}^{2}}$ & $h_{r}+h_{d}[p]$ \\
\hline$c\left[p_{0}\right]$ & $h_{r}+h_{d}[p]$ & $h_{r}+h_{d}[p]$ \\
\hline
\end{tabular}

Table 1. Elastohydrodynamic homogenized coefficients

Let us remark that the smooth rigid gap is given by

$$
h_{r}(x)=h_{0}+\frac{x_{1}^{2}+x_{2}^{2}}{2} .
$$




\subsection{Case 1: transverse roughness tests}

Although numerical tests have been performed for different spatial meshes in order to validate the convergence of the methods, we just present the results corresponding to $\Delta x_{1}=0.02$ and $\Delta x_{2}=0.133$ so that we have 44400 triangles and 22575 vertices. Furthermore, the artificial time step in the characteristics method [5, 14], $\Delta t=\Delta x_{1}$; the Bermudez-Moreno parameters are $\omega=1$ and $\lambda=1 /(2 \omega)$; the stopping test in all algorithms is equal to $\delta=10^{-5}$ (corresponding to the relative error in the discrete $L^{2}$ norm between two iterations).

The computer results illustrate the convergences stated in previous sections. First in Fig.2, we show the strong convergence of the pressure to the homogenized one, when $\varepsilon$ tends to 0 , by plotting the cuts at $x_{2}^{0}=0$, for different values of $\varepsilon$ and the homogenized solution. In FIG.3, the homogenized pressure over the whole domain is presented. In FIG.4 and 5, analogous plots for the deformation are displayed to illustrate the convergence and the homogenized distribution. Finally, in Fig.6 and 7 , the results for the saturation are shown. To be noticed is the weak convergence of the saturation, linked to the existence of oscillations which are not damped, unlike the pressure and deformation. We can also notice that the deformation oscillations are damped very easily (when compared to the pressure oscillations). This is due to the regularizing effects of the Hertz kernel (which is a convolution-like operator).

\subsection{Case 2: longitudinal roughness tests}

In this case, we present the results corresponding to $\Delta x_{1}=0.06$ and $\Delta x_{2}=0.02$ so that we have 40000 triangles and 20301 vertices.

The computer results illustrate the convergences stated in previous sections. First in Fig.8, we show the strong convergence of the pressure to the homogenized one, when $\varepsilon$ tends to 0 , by plotting the cuts at $x_{1}^{0}=-2.5$ (corresponding to the maximum homogenized pressure), for different values of $\varepsilon$ and the homogenized solution. In FIG.9, the homogenized pressure over the whole domain is presented. In FIG.10 and 11, the homogenized deformation and saturation over the whole domain are presented. Notice that at $x_{1}^{0}=-2.5$, all saturations are identically 1 .

\subsection{Influence of the elastic contribution over the roughness effects}

We compare the results, in a transverse roughness case, between hydrodynamic and elastohydrodynamic configurations.

In FIG.12 and 13, we present the pressure and saturation profiles in the following cases: $l_{1}=2, l_{2}=2, h_{0}=0.6$ and

- hydrodynamic smooth solution

- hydrodynamic homogenized solution

- elastohydrodynamic smooth solution

- elastohydrodynamic homogenized solution

$$
\begin{aligned}
& \text { : } \quad \alpha_{1}=0, \quad \alpha_{2}=0, \quad k \equiv 0, \\
& \alpha_{1}=0.85 h_{0}, \quad \alpha_{2}=0, \quad k \equiv 0, \\
& \alpha_{1}=0, \quad \alpha_{2}=0, \quad k \neq 0, \\
& \alpha_{1}=0.85 h_{0}, \quad \alpha_{2}=0, \quad k \neq 0 .
\end{aligned}
$$




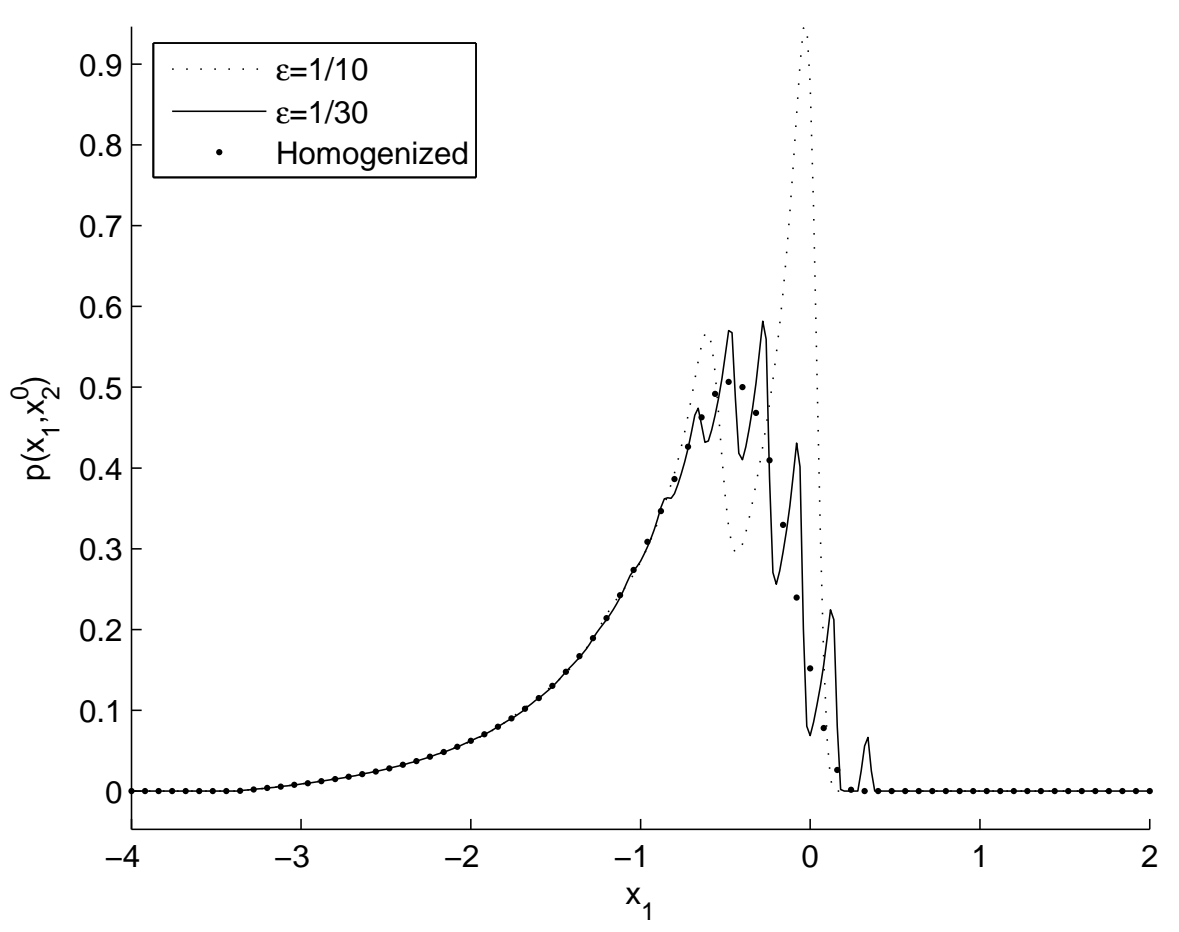

Fig. 2. Elastohydrodynamic pressure at $x_{2}^{0}=0$ (transverse roughness)

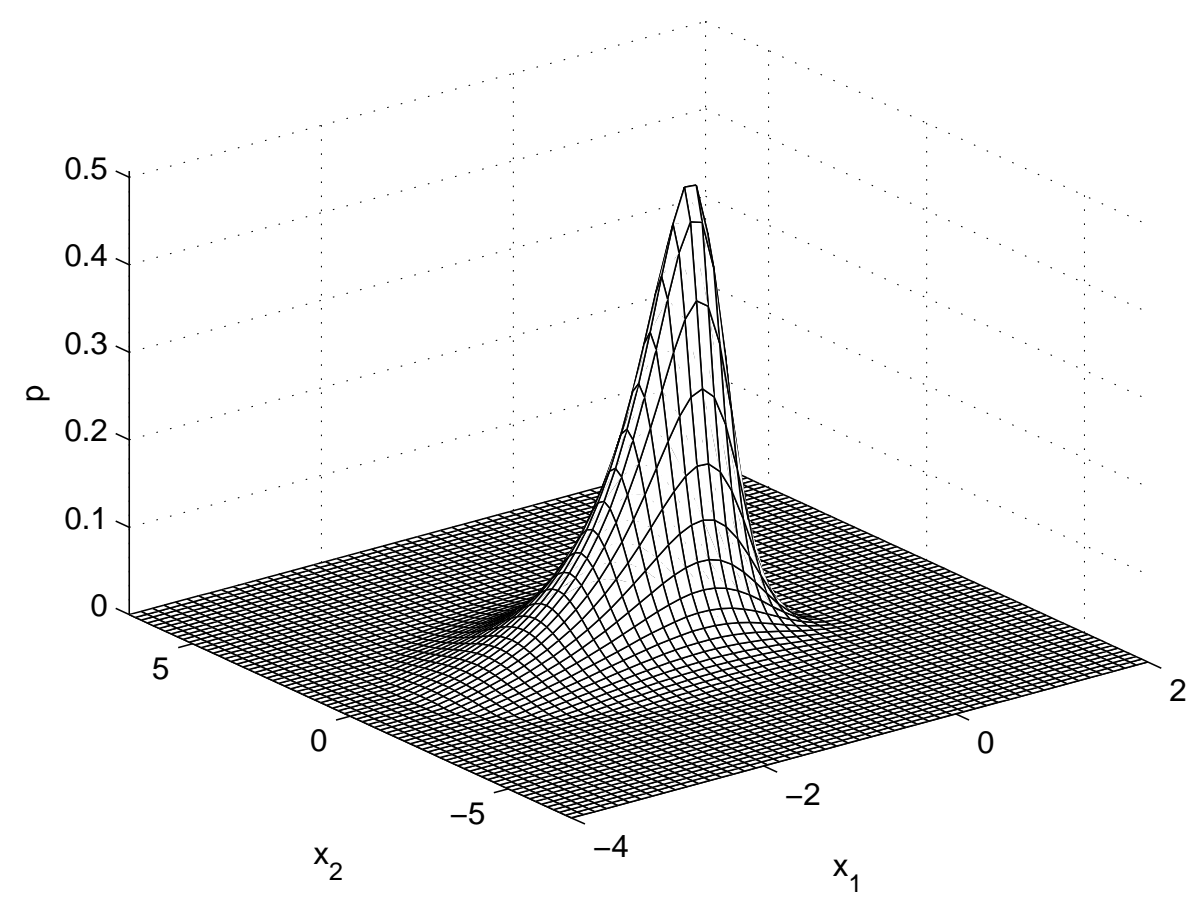

Fig. 3. Elastohydrodynamic homogenized pressure (transverse roughness) 


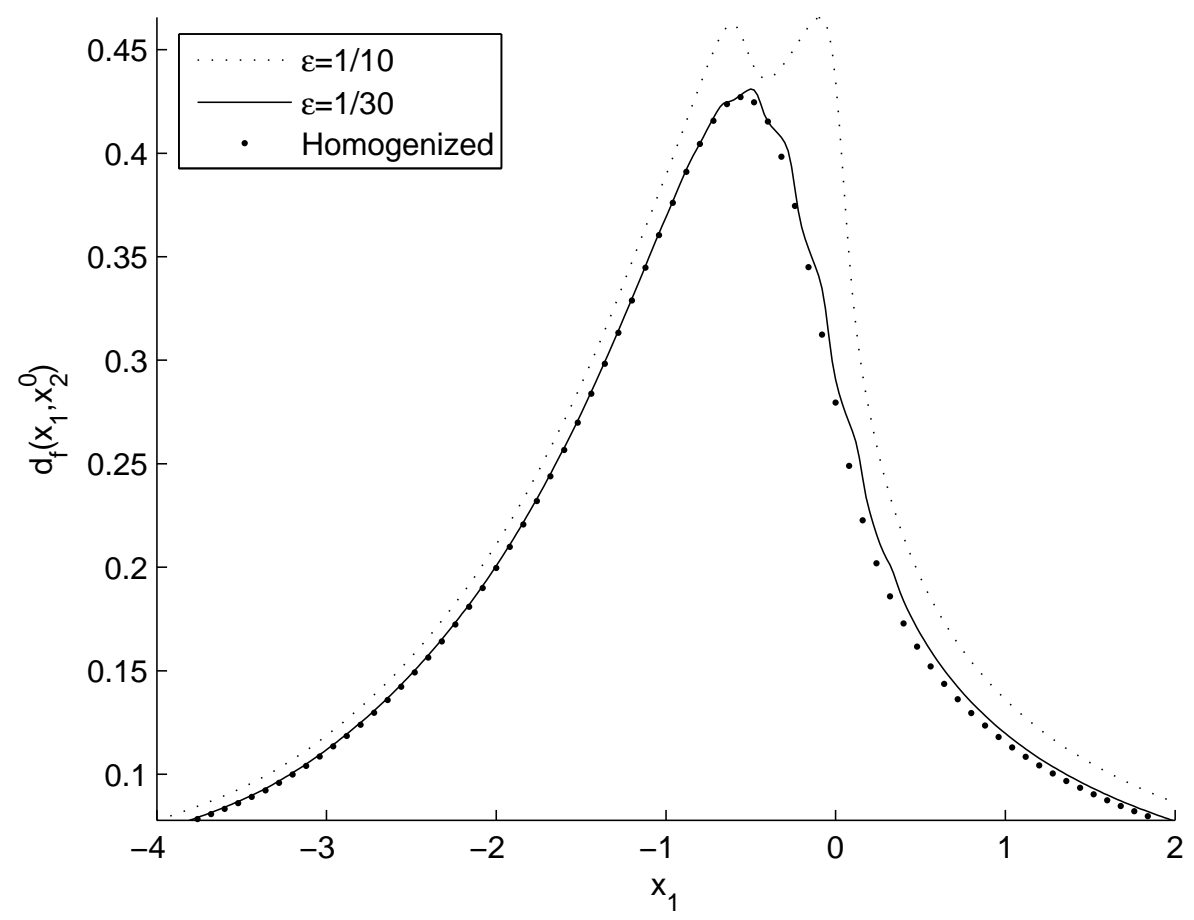

Fig. 4. Elastohydrodynamic deformation at $x_{2}^{0}=0$ (transverse roughness)

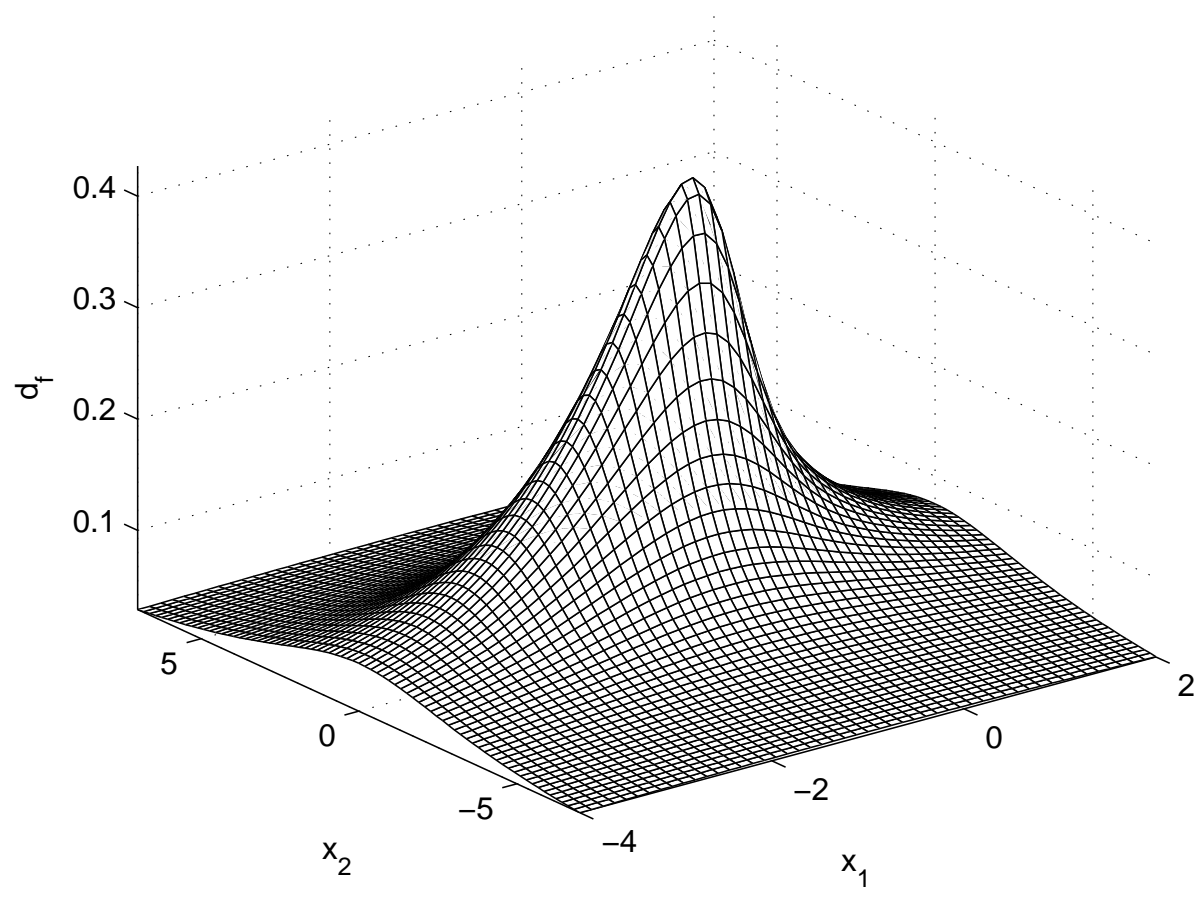

Fig. 5. Elastohydrodynamic homogenized pressure (transverse roughness) 


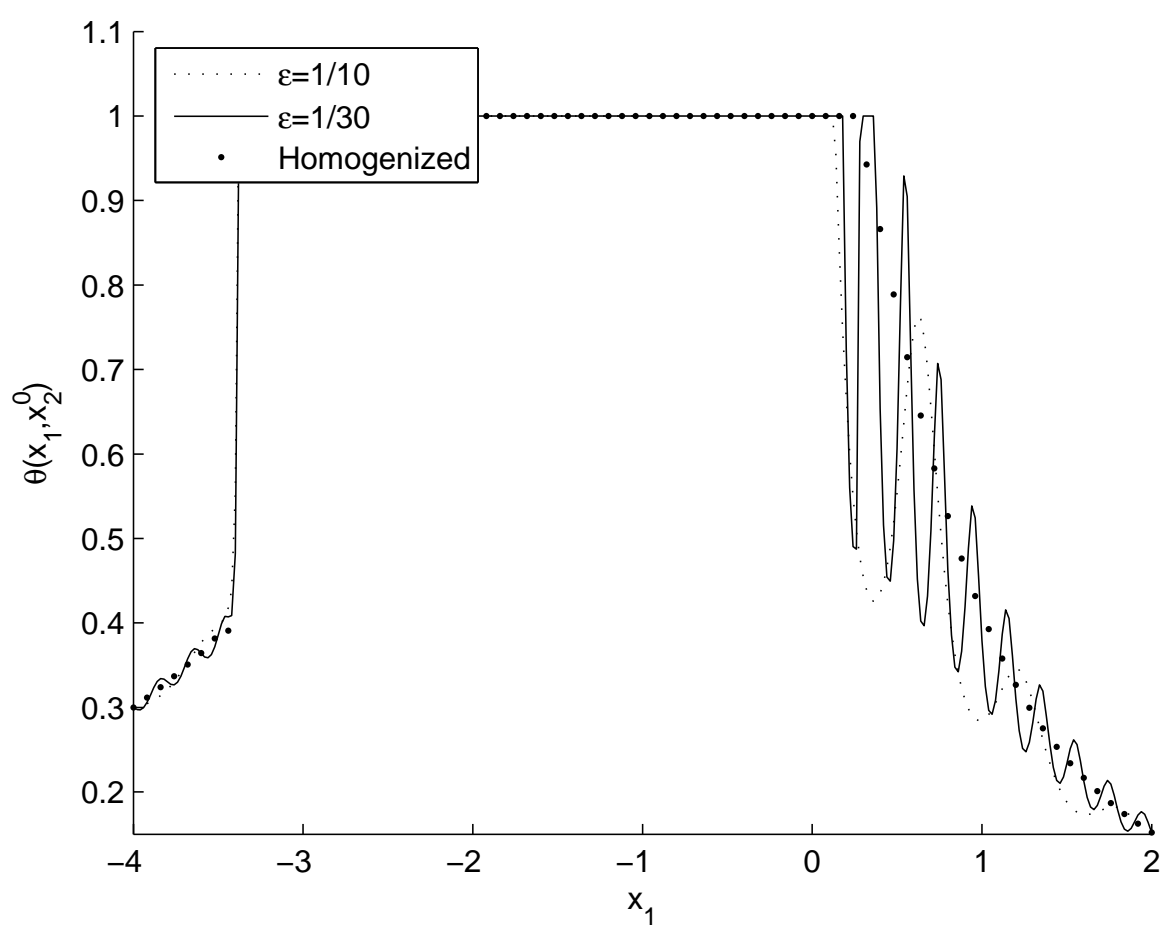

Fig. 6. Elastohydrodynamic saturation at $x_{2}^{0}=0$ (transverse roughness)

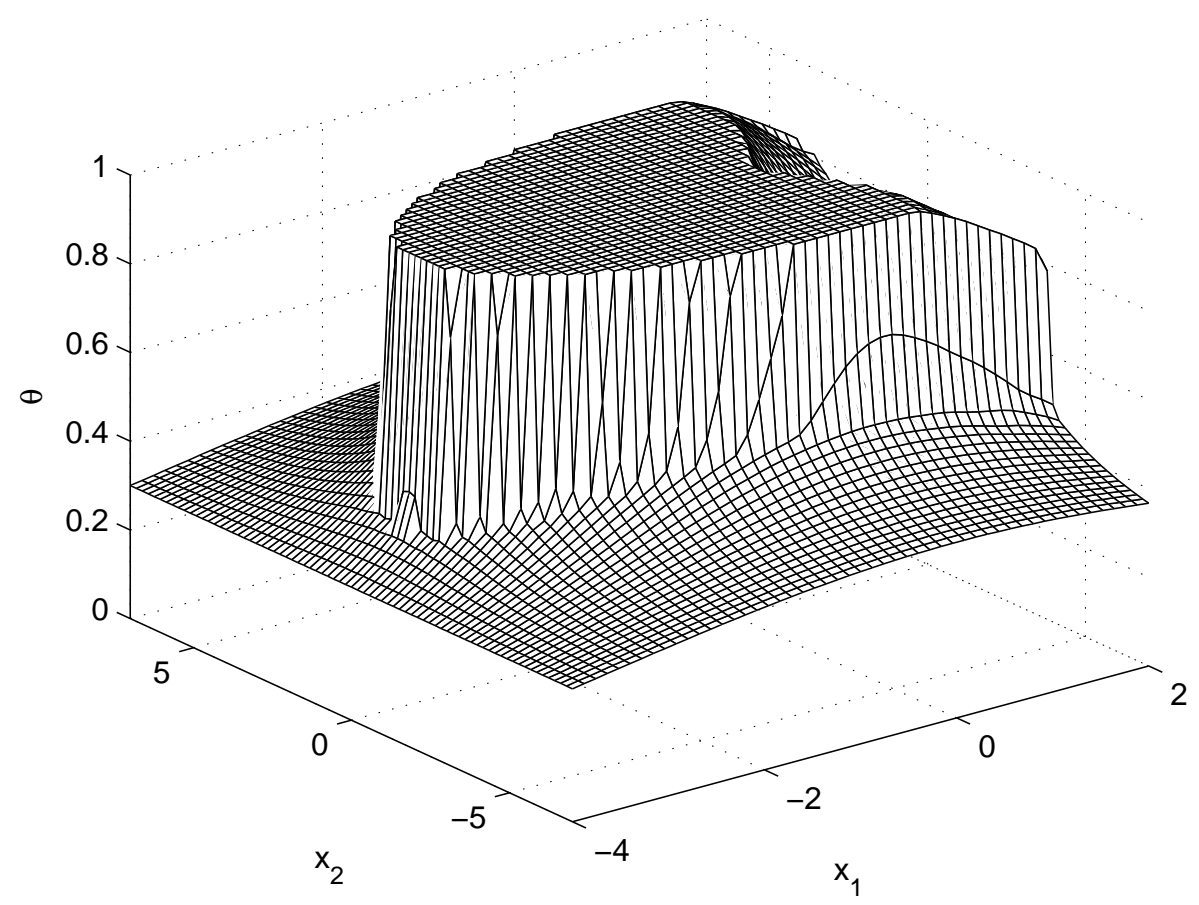

Fig. 7. Elastohydrodynamic homogenized saturation (transverse roughness) 


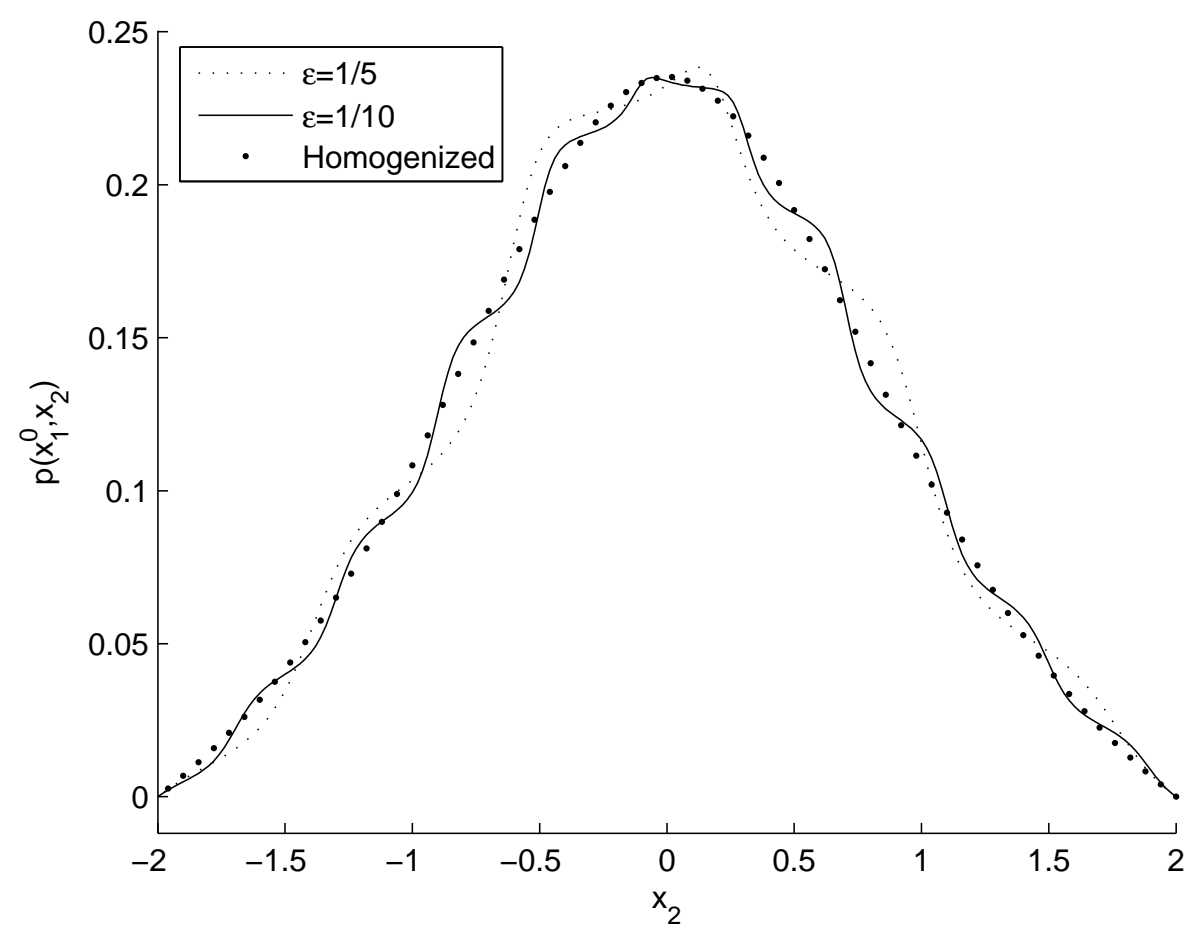

Fig. 8. Elastohydrodynamic pressure at $x_{1}^{0}=-2.5$ (longitudinal roughness)

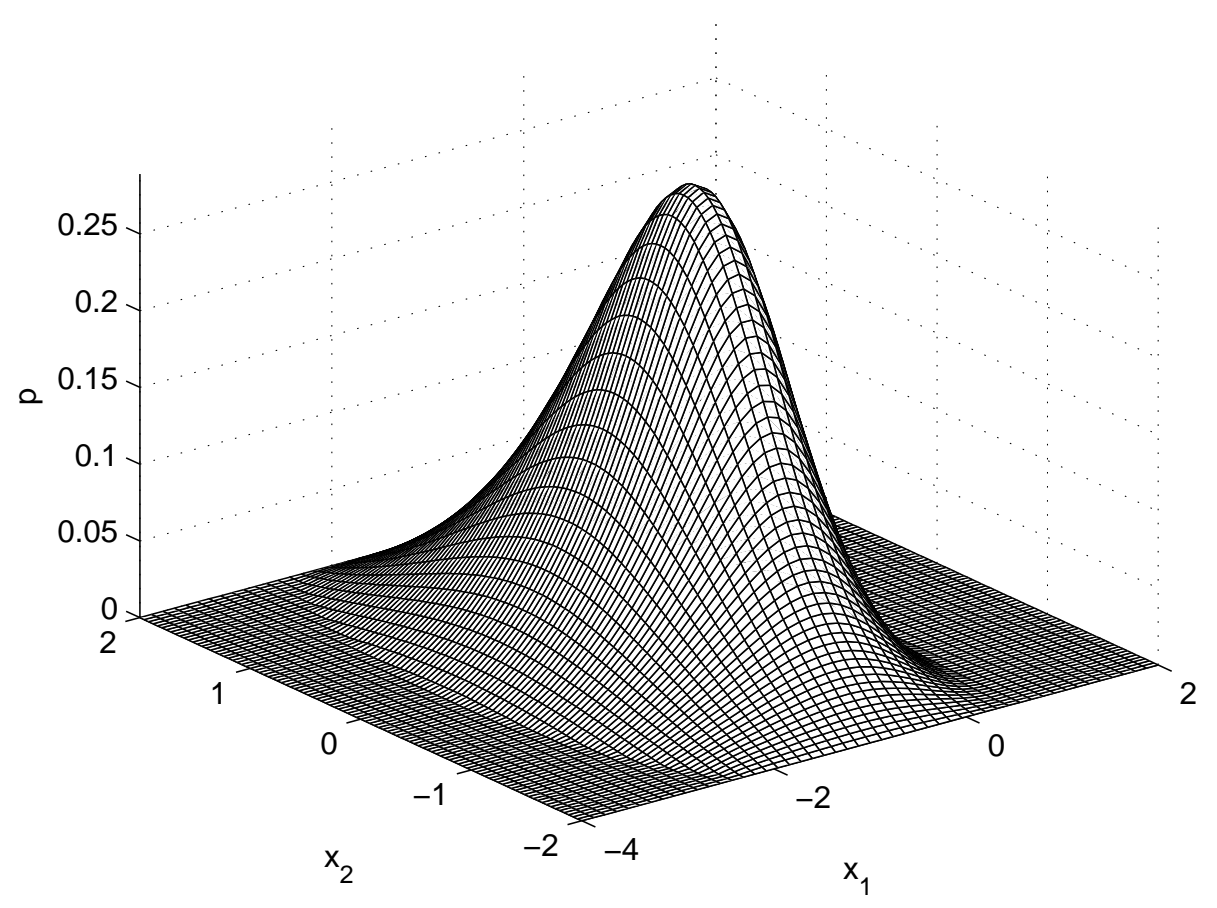

Fig. 9. Elastohydrodynamic homogenized pressure (longitudinal roughness) 


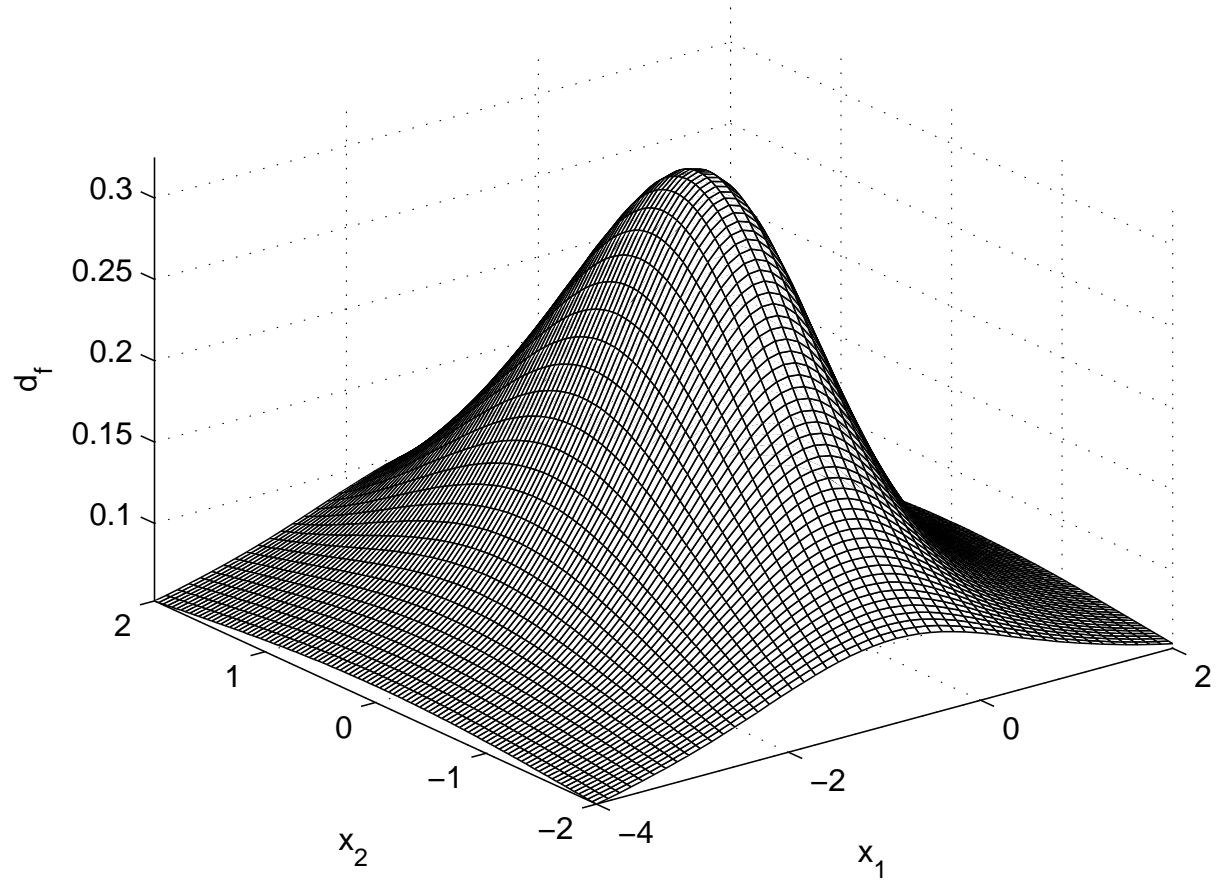

Fig. 10. Elastohydrodynamic homogenized deformation (longitudinal roughness)

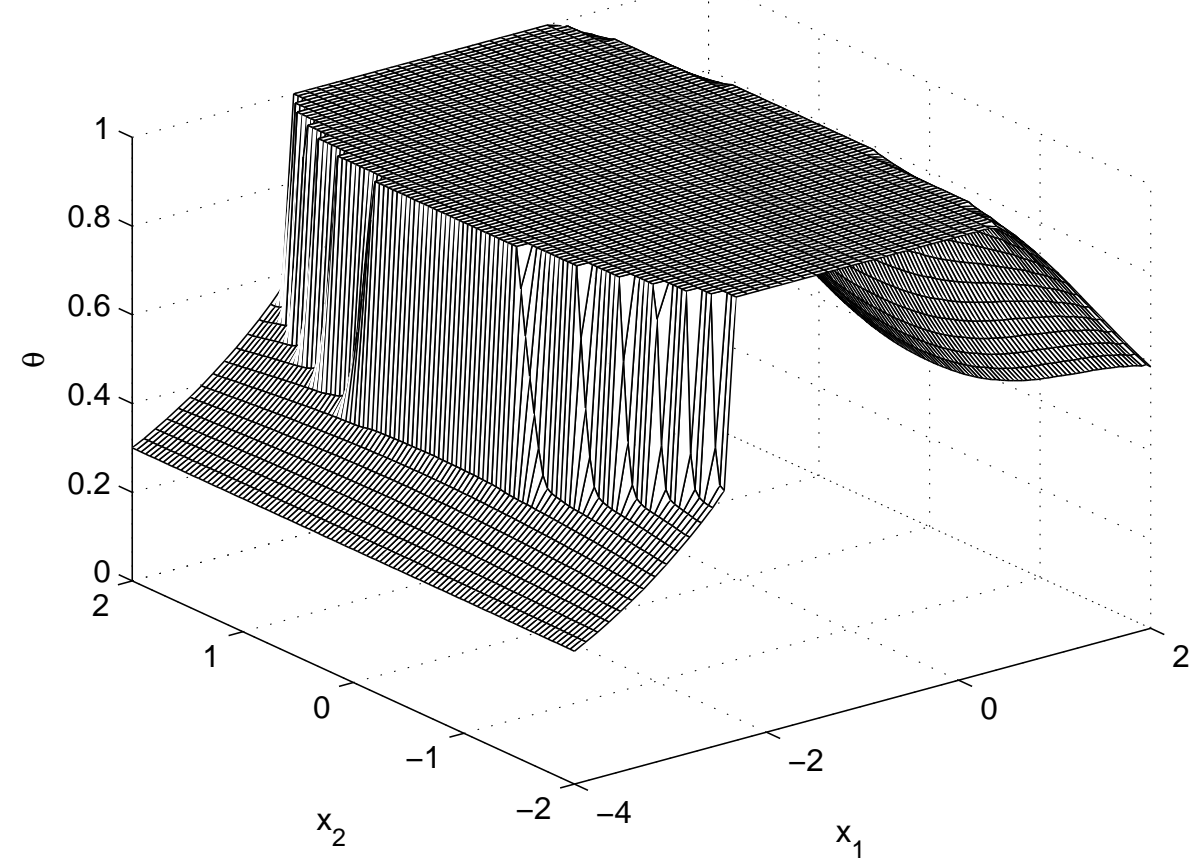

Fig. 11. Elastohydrodynamic homogenized saturation (longitudinal roughness) 
The figures correspond to a fixed $x_{2}^{0}=0$. It allows us to see the influence of the roughness over the pressure distribution in each case (hydrodynamic or elastohydrodynamic), but also the importance of the roughness over the maximum pressure. The roughness effects are clearly more important in the hydrodynamic regime than in the elastohydrodynamic one.

\section{References}

[1] G. Allaire. Homogenization and two-scale convergence. SIAM J. Math. Anal., 23(6):1482-1518, 1992.

[2] C. Barus. Isothermals, isopiestics and isometrics relative to viscosity. American Journal of Science, 45:87-96, 1893.

[3] G. Bayada and M. Chambat. Sur quelques modélisations de la zone de cavitation en lubrification hydrodynamique. J. Méc. Théor. Appl., 5(5):703-729, 1986.

[4] G. Bayada and M. Chambat. The transition between the Stokes equations and the Reynolds equation: a mathematical proof. Appl. Math. Optim., 14(1):7393, 1986.

[5] G. Bayada, M. Chambat, and C. Vázquez. Characteristics method for the formulation and computation of a free boundary cavitation problem. J. Comput. Appl. Math., 98(2):191-212, 1998.

[6] G. Bayada, B. Cid, and C. Vázquez. Two-scale homogenization study of a Reynolds-rod elastohydrodynamic model. Math. Models Methods Appl. Sci., 13(2):259-293, 2003.

[7] G. Bayada, M. El Alaoui Talibi, and C. Vázquez. Existence of solutions for elastohydrodynamic piezoviscous lubrication problems with a new model of cavitation. European J. Appl. Math., 7(1):63-73, 1996.

[8] G. Bayada and J.-B Faure. A double-scale analysis approach of the Reynolds roughness. Comments and application to the journal bearing. ASME J. Tribol., 111:323-330, 1989.

[9] G. Bayada, S. Martin, and C. Vázquez. Two-scale homogenization of a hydrodynamic Elrod-Adams model. Asymptot. Anal., 44(1-2):75-110, 2005.

[10] H. Brezis. Analyse fonctionnelle. Collection Mathématiques Appliquées pour la Maîtrise. [Collection of Applied Mathematics for the Master's Degree]. Masson, Paris, 1983. Théorie et applications. [Theory and applications].

[11] D. Cioranescu, A. Damlamian, and G. Griso. Periodic unfolding and homogenization. C. R. Math. Acad. Sci. Paris, 335(1):99-104, 2002.

[12] D. Dowson and G. R. Higginson. Elastohydrodynamic lubrication. Pergamon Press, Oxford, Great Britain, 1977.

[13] J. Durany, G. García, and C. Vázquez. A mixed Dirichlet-Neumann problem for a nonlinear Reynolds equation in elastohydrodynamic piezoviscous lubrication. Proc. Edinburgh Math. Soc. (2), 39(1):151-162, 1996. 


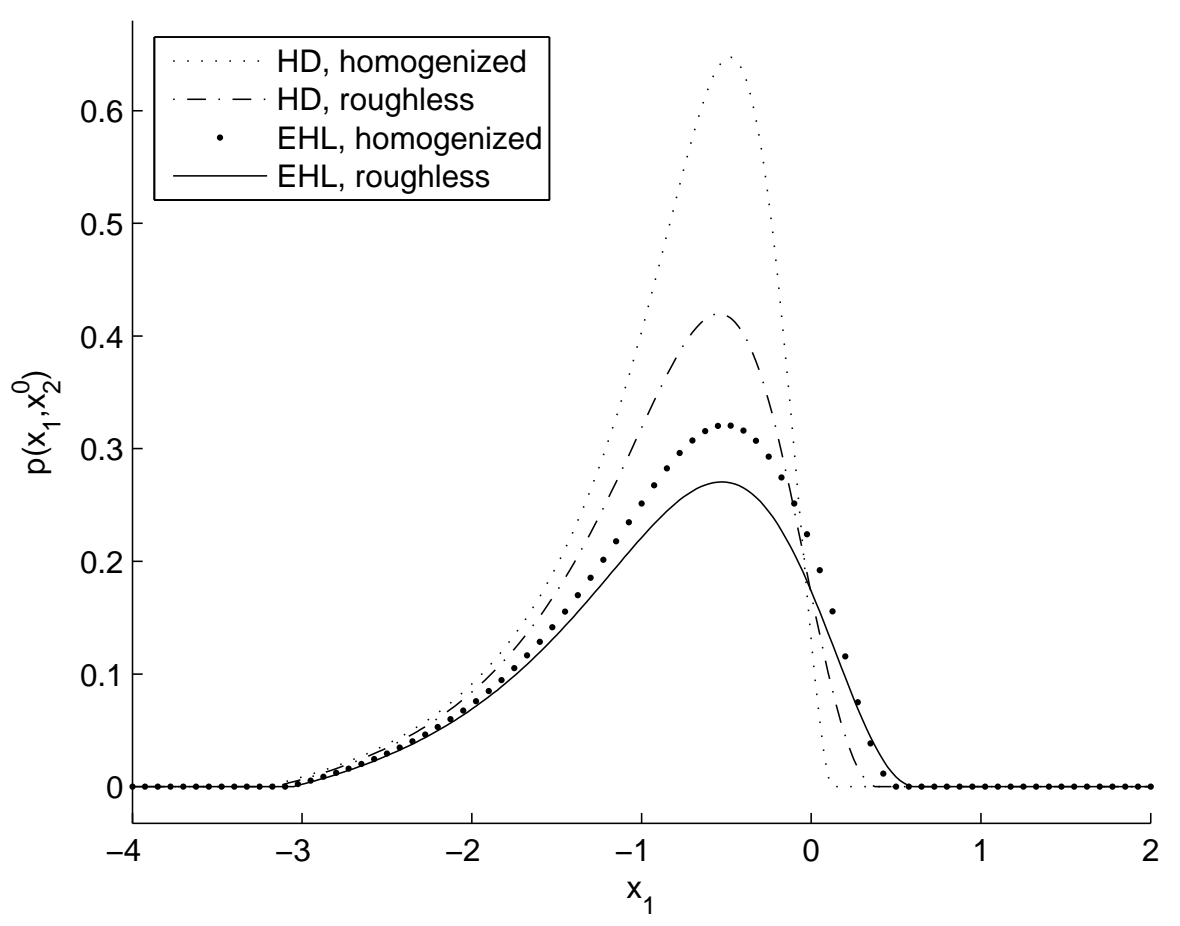

Fig. 12. Roughness effects over EHL and hydrodynamic pressures

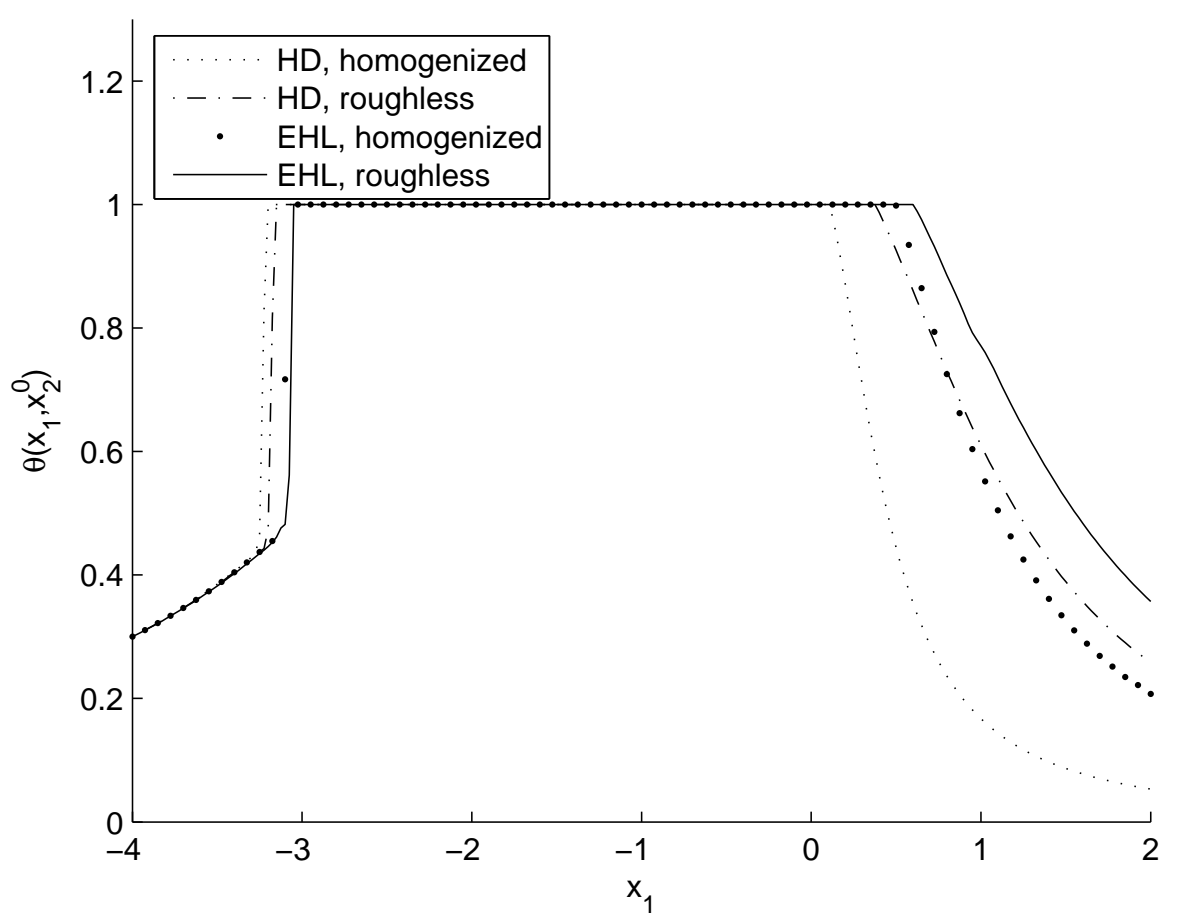

Fig. 13. Roughness effects over EHL and hydrodynamic saturations 


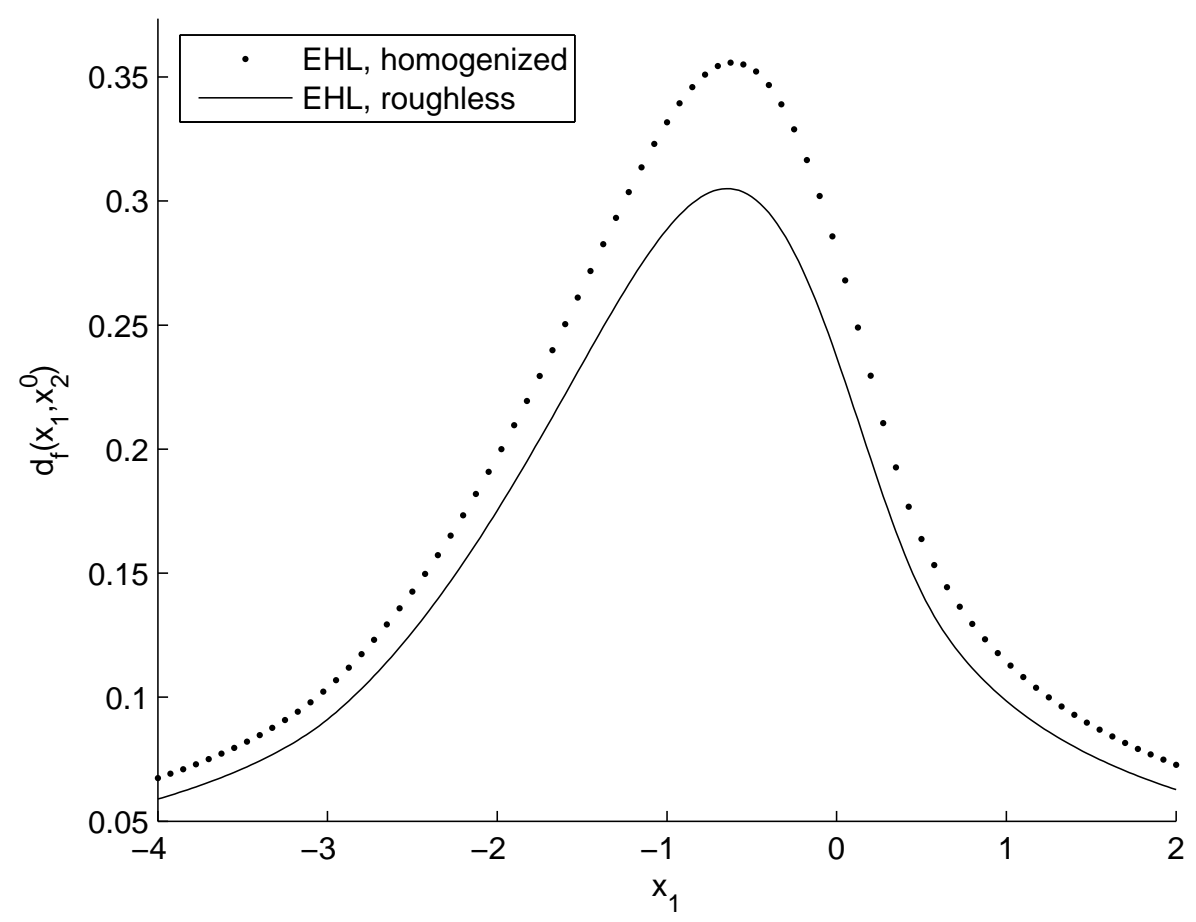

Fig. 14. Influence of the rougness effects on the deformation

[14] J. Durany, G. García, and C. Vázquez. Numerical computation of free boundary problems in elastohydrodynamic lubrication. Appl. Math. Modelling, 20:104-113, 1996.

[15] J. Durany, G. García, and C. Vázquez. Numerical simulation of a lubricated Hertzian contact problem under imposed load. Finite Elem. Anal. Des., 38(7):645-658, 2002.

[16] H. G. Elrod and M. L. Adams. A computer program for cavitation. Cavitation and related phenomena in lubrication - Proceedings - Mech. Eng. Publ. ltd, pages $37-42,1975$.

[17] D. Goeleven and V. H. Nguyen. On the one-dimensional nonlinear elastohydrodynamic lubrication. Bull. Austral. Math. Soc., 50(3):353-372, 1994.

[18] C. J. Hooke. The behaviour of low-amplitude surface roughness under line contacts. Proc. Instn. Mech. Engrs., 213:275-285, 1998.

[19] B. Hu. A quasi-variational inequality arising in elastohydrodynamics. SIAM J. Math. Anal., 21(1):18-36, 1990.

[20] M. Jai. Homogenization and two-scale convergence of the compressible Reynolds lubrication equation modelling the flying characteristics of a rough 
magnetic head over a rough rigid-disk surface. RAIRO Modél. Math. Anal. Numér., 29(2):199-233, 1995.

[21] D. Lukkassen, G. Nguetseng, and P. Wall. Two-scale convergence. Int. J. Pure Appl. Math., 2(1):35-86, 2002.

[22] G. Nguetseng. A general convergence result for a functional related to the theory of homogenization. SIAM J. Math. Anal., 20(3):608-623, 1989.

[23] J. T. Oden and S. R. Wu. Existence of solutions to the Reynolds' equation of elastohydrodynamic lubrication. Internat. J. Engrg. Sci., 23(2):207-215, 1985.

[24] O. Reynolds. On the theory of lubrication and its application to Mr Beauchamp tower's experiments, including an experimental determination of the viscosity of olive oil. Phil. Trans. Roy. Soc., A 117:157-234, 1886.

[25] J.-F. Rodrigues. Remarks on the Reynolds problem of elastohydrodynamic lubrication. European J. Appl. Math., 4(1):83-96, 1993. 